\title{
Floristic and phytosociology in dense "terra firme" rainforest in the Belo Monte Hydroelectric Plant influence area, Pará, Brazil
}

\author{
Lemos, DAN..$^{a, b *}$, Ferreira, BGA. ${ }^{c}$, Siqueira, JDP. ${ }^{b, c}$, Oliveira, MM. ${ }^{d, b}$ and Ferreira, AM..$^{b, c}$ \\ ${ }^{a}$ University of La Coruña - UDC, Rúa da Maestranza, 9, 15001, La Coruña, Spain \\ 'bTCP Engenharia de Projetos Ltda., Rua Euzébio da Motta, 450, Juvevê, CEP 80530-260, Curitiba, PR, Brazil \\ 'Universide Federal do Paraná - UFPR, Rua dos Funcionários, 1540, Juvevê, CEP 80035-050, Curitiba, PR, Brazil \\ dInstituto Nacional de Pesquisas da Amazônia - INPA, Av. André Araújo, 2936, Aleixo, \\ CEP 69060-001, Manaus, AM, Brazil \\ *e-mail: nevesdeb@hotmail.com
}

Received: May 5, 2014 - Accepted: March 20, 2015 - Distributed: August 31, 2015

(With 3 figures)

\begin{abstract}
The objective of the present study was to characterise the floristic and phytosociological composition on a stretch of dense "Terra Firme" rainforest located in the Belo Monte hydroelectric plant area of influence, located in the state of Pará, Brazil. All trees with DAP $>10 \mathrm{~cm}$ situated in 75 permanent plots of 1 ha were inventoried. 27,126 individuals trees (361 ind.ha-1), distributed in 59 botanical families, comprising 481 species were observed. The families with the largest number of species were Fabaceae (94), Araceae (65) and Arecaceae (43), comprising 43.7\% of total species. The species Alexa grandiflora (4.41), Cenostigma tocantinum (2.50) and Bertholletia excelsa (2.28) showed the highest importance values (IV). The ten species with greater IV are concentrated (22\%). The forest community has high species richness and can be classified as diverse age trees, heterogeneous and of medium conservation condition.
\end{abstract}

Keywords: floristic survey, diversity, amazon species, rain forest.

\section{Florística e fitossociologia de um trecho de Floresta ombrófila densa de terra firme na área de influência da Usina Hidrelétrica Belo Monte, Pará, Brasil}

\begin{abstract}
Resumo
O presente estudo teve como objetivo caracterizar a composição florística e fitossociológica de um trecho de Floresta Ombrófila Densa de terra firme na área de influência da Usina Hidrelétrica Belo Monte, Pará, Brasil. Foram inventariadas todas as árvores com DAP $>10 \mathrm{~cm}$ em 75 parcelas permanentes de 1 ha. Foram observados 27.126 indivíduos arbóreos (361 ind.ha-1), distribuídos em 59 famílias botânicas, perfazendo 481 espécies. As famílias com maior número de espécies foram Fabaceae (94), Araceae (65) e Arecaceae (43), perfazendo 43,7\% do total de espécies amostradas. As espécies Alexa grandiflora (4,41), Cenostigma tocantinum $(2,50)$, Bertholletia excelsa $(2,28)$, apresentaram os maiores valores de importância (VI), sendo que nas dez espécies com maiores IV, estão concentrados $22 \%$. A comunidade florestal apresenta elevada riqueza florística e pode ser classificada como multiâneas, heterogêneas e com médio estado de conservação.
\end{abstract}

Palavra-chave: levantamento florístico, diversidade, espécies amazônicas, floresta tropical.

\section{Introduction}

Phytosociological studies have special interest in tropical forests due to the wide variety of patterns and processes related to their diversity. Tropical forests have gained importance in recent decades not only for their natural aspects but also for their social and economic aspects, leading to discussions in the scientific/ecological and social context (Lima et al., 2012). However, there are just a few studies on the floristic, structural and dynamics composition of these ecosystems. The consequences are major knowledge gaps in geographical terms, especially because there are found many floristically distinct forest formations in regions of apparently homogeneous vegetation (Hopkins, 2007; Lima et al., 2012).

Among the tropical forests, dense rainforest is considered one of the most important 'hot spots' for its greatest richness and diversity (Laurance, 2008). Several studies have shown that plateau and slope forest environments exhibit high species diversity, represented by few individuals of each species and highly variable values of diversity and similarity (Lima Filho et al., 2004).

Information on forest structure and floristic composition obtained through forest inventories are among the main 
available tools to assess the potential of a forest and to choose the forest management strategies. In this context, the use of phytosociological criteria increases knowledge of species that belong to significant stretches of tropical forests. They enable the planning and execution of appropriate strategies for biodiversity conservation and development of more efficient environmental practices. Thus, the present study aimed to characterise the floristic and phytosociological composition on a stretch of dense "terra firme" rainforest situated in the Belo Monte hydroelectric plant area of influence, in the state of Pará, Brazil.

\section{Material and Methods}

The study site is located at a place called Volta Grande do Rio Xingu. It is situated in northern Brazil, at the Xingu River, a tributary on the right bank of the Amazon River in Pará state $\left(03^{\circ} 26^{\prime}\right.$ south latitude e $51^{\circ} 56^{\prime}$ west longitude). Volta Grande do Rio Xingu is situated between the town of Altamira and the locations of Belo Monte and Belo Monte do Pontal, respectively located in the cities of Vitória do Xingu and Anapu. Forest types under study are characterised as Dense Rain Forest (FOD) "terra firme". It is located in the direct and indirect area of influence of the Belo Monte Hydroelectric Plant (UHE Belo Monte). (Brasil, 2009) (Figure 1).

The region offers a variety of soil types like neossolos fluvic and lithic, ultisols, latosols, cambisoils, Haplic Gleysols and Nitosols Haplic (Brasil, 2009). According to Koppen's classification, the area's climate is tropical with monsoon rainfall. There is a short duration dry season which corresponds to (Am) classification and a humid tropical with a slightly pronounced dry season with very high rainfall (Amw) (Brasil, 2009).

Sampling and data collection followed RAPELD's methodology described by the authors Magnusson et al.

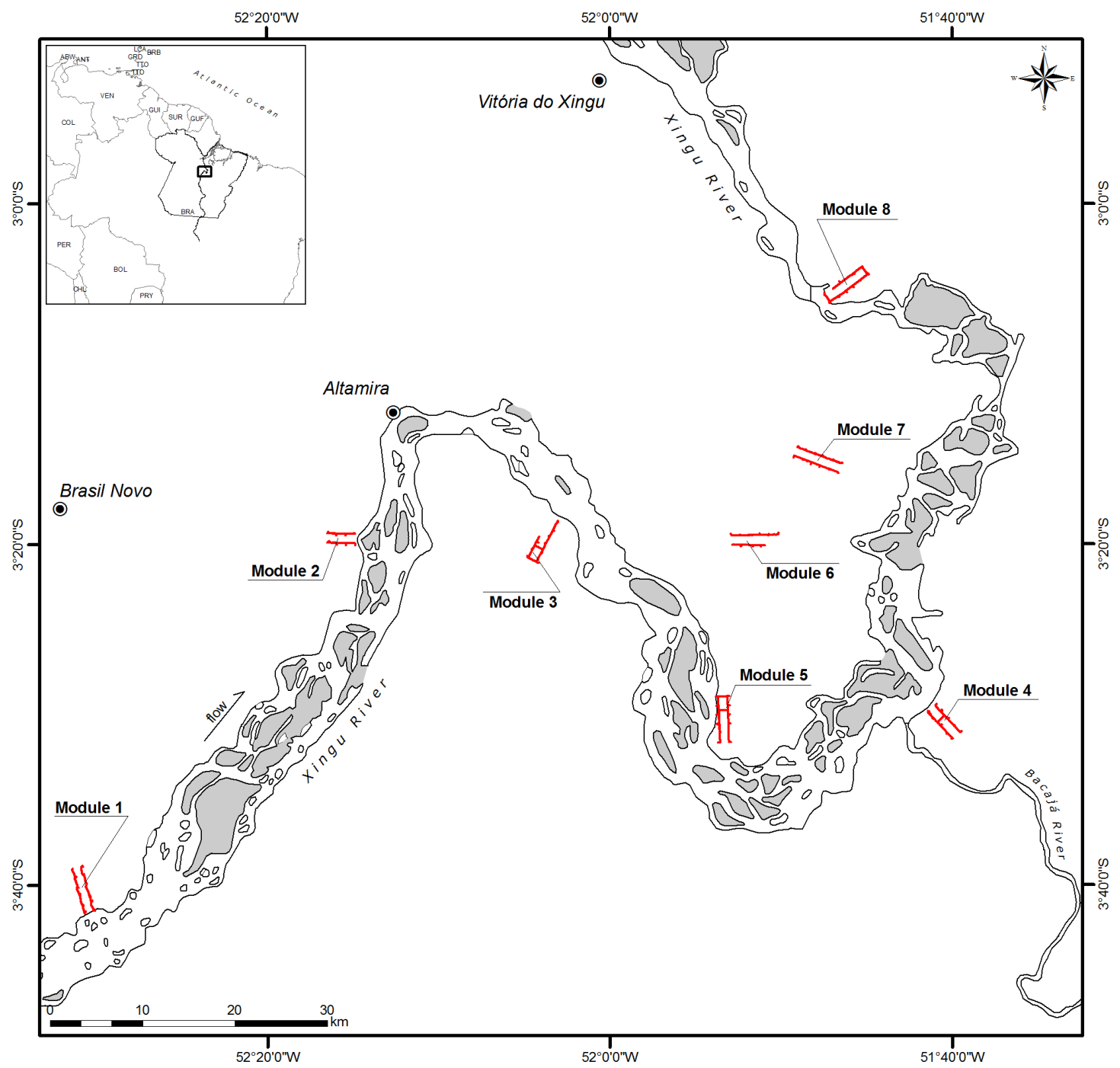

Figure 1. Belo Monte hydroelectric plant's area of influence and location of RAPELD sampling modules. 
(2005), which merges rapid assessments with long-term studies.

The sampling units were placed in the four compartments of Belo Monte as shown in Figure 1:

1) Xingu Reservoir (Modules 2/3),

2) Stretch of Low Flow (Modules 4/5),

3) Intermediate Reservoir (Modules 6/7), and

4) Stretch downstream of the main powerhouse (no intervention) (Modules 1/8).

Each RAPELD module is a $5 \mathrm{~km}^{2}$ area where two $5 \mathrm{~km}$ parallel transects were installed, $1 \mathrm{~km}$ apart, where 12 permanent $250 \mathrm{~m}$ plots ( 6 in each transect) are installed. Those plots are $40 \mathrm{~m}$ wide, perpendicular to the transects and with a central axis following the contour line. However, from the 96 plots initially planned and required by the methodology ( 8 Modules x 12 plots per Module), it was not possible to install 13 due to land problems, thus making a total of 83 sampling units. From those, 75 were distributed in the dense "terra firme" rainforests (FOD). They were distributed in 8 RAPELD Modules. Floristic and phytosociological estimators were performed by sampling 75 permanent plots distributed in eight RAPELD modules. The plots were marked at $10 \mathrm{~m}$ from the transect in order to reduce the edge effect from the transect's opening. Each parcel was $250 \mathrm{~m}$ long and $40 \mathrm{~m}$ wide $(20 \mathrm{~m}$ on each side of the centerline) resulting in 1 ha per parcel. Individual trees sampled were at least $10 \mathrm{~cm}$ breast height diameter (diameter measured at $1.30 \mathrm{~m}$ high).

To characterise the floristic composition of Belo Monte's area of influence, the growth habits according to Raunkiaer classification (Raunkiaer, 1934) were considered. This was adapted to Brazilian conditions (IBGE, 2012) as follows: trees, shrubs, grass, holoepiphytes, hemiepiphytes, lianas and palms. Additionally, species richness was analysed based on the number of species and plant families found in this classification.

Data collection was carried out between March and December 2014. The dendometric quantified variables were breast height circumference (circumference at $1.30 \mathrm{~m}$ height above the ground) and height. Numbered metallic plates were used to identify the circumference measurements.

Tree species botanical identification occurred from the botanical collection of all sampled individuals. An average of 6 samples from each was collected. The botanical material was classified by family, genus and species. The reference was Angiosperm Phylogeny Group III. The unicata and a duplicate were deposited at the Emilio Goeldi Museum (MPEG); other duplicates were kept for the Federal University of Pará (UFPA) Campus Altamira and for the Botanical Museum of Curitiba City - Herbarium MBM collections.

To characterise the fragment's horizontal structure, the following phytosociological parameters were analysed according to Müller-Dombois and Ellemberg (1974): absolute density (AD), relative density (RD), absolute frequency $(\mathrm{AF})$, relative frequency $(\mathrm{RF})$, absolute dominance (ADo), relative dominance (RDo), coverage value (CV) and importance value (IV). The parameters were calculated at Microsoft Excel for Windows software 2007. Sample sufficiency was calculated by linear regression model with plateau response (REGRELRP), performed by the statistical program R (R Development Core Team, 2008).

\section{Results}

\subsection{Floristic composition}

The sampled area revealed the presence of 726 species in 297 genera and 79 botanical families for the eight RAPELD modules. Regarding species richness, families with greater representation were: Fabaceae (94 species) followed by the Araceae (65), Arecaceae (43), Sapotaceae (37), Moraceae (30), Orchidaceae (29), Marantaceae (26), Lauraceae (25), Lecythidaceae (21) and Annonaceae (21).

Rare families are considered those for which only one species was found. Very rare are those with a single species and a single individual found. Overall $26 \%$ of families can be classified as rare and $10 \%$ as very rare. The Fabaceae family is the botanical family with the greatest abundance.

Analysing grass growth habit species, holoepiphytes, hemiepiphytes, lianas and palms, it is noted that most species are concentrated on frequent and abundant classes (Figure 2). The following species can be highlighted: Anthurium ernestii Engl., Dieffenbachia humilis Poepp., Epidendrum rigidum Jacq., Philodendron mellinonii Brongn. ex Regel, Philodendron pectinathum, Philodendron solimoesense A.C.Sm., Bactris acanthocarpa Barb.Rodr., Bactris brongniartii Mart., Chamaedorea pinnatifrons (Jacq.) Oerst., Syagrus inajai (Spruce) Becc., Aechmea longifolia (Rudge) L.B.Sm. \& M.A.Spencer, Pereskia aff. aculeata Mill., Rhipsalis bacifera (J.M.Muell.) Stearn, Heliconia densiflora var. densiflora Verl., Goeppertia fragilis (Gleason) Borchs. \& S.Suárez, Brassia lanceana Lindl., Epidendrum cristatum Ruiz \& Pav., Epidendrum schlechterianum Ames, Lockhartia imbricata (Lam.) Hoehne, Ornithocephalus bicornis Lindl., Pleurothallis yauaperyensis Barb.Rodr., Sobralia sessilis Lindl..

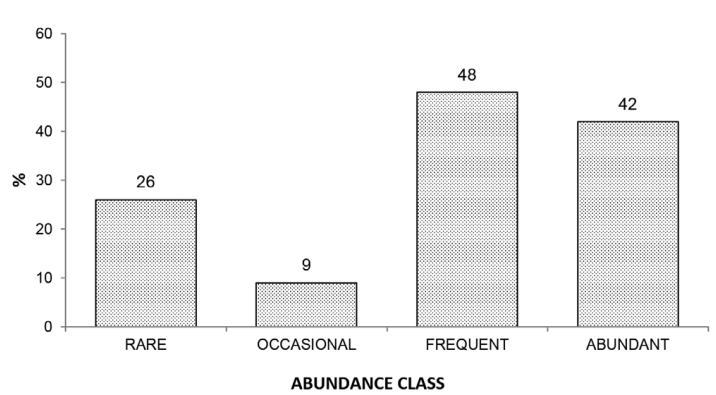

Figure 2. Species of grass growth habit, holoepiphytes, hemiepiphytes, lianas and palms percentages according to the class of abundance for dense "terra firme" rainforest located in Belo Monte Hydroelectric Plant's area of influence, situated in Pará, Brazil. 


\subsection{Phytosociologycal composition}

A total of 27,126 trees (361 ind.ha ${ }^{-1}$ ) were identified. They are distributed in 59 families and 481 species. Species sampled on the 75 plots distributed at 8 RAPELD modules which are situated at UHE Belo Monte's influence area are presented in alphabetical order in Table 1.

The highest absolute density species were: Cecropia obtusa (13.33), Theobroma speciosum (13.07), Alexa grandiflora (11.87), Cenostigma tocantinum (11.13), Guapira venosa (10.92), Vouacapoua americana (8.68), Mabea speciosa (7.03), Inga edulis (6.77), Cecropia membranacea (6.27) and Jacaranda copaia (6.15) (Table 1).
Overall, 3.4\% are considered low occurrence species. In other words 16 species are represented by a single individual, amongst which can be mentioned Anacardium occidentale, Campsiandra laurifolia, Cariniana micranta, Copaifera multijuga and Dussia discolor. Among the species with higher relative dominance, Alexa grandiflora (8.82\%), Bertholletia excelsa (5.39\%), Cenostigma tocantinum (4.01\%), Vouacapoua americana (3.65\%), Cecropia obtusa $(2.42 \%)$ and Inga edulis $(2.01 \%)$ stand out.

The average basal area was $13.96 \mathrm{~m}^{2} / \mathrm{ha}$. Basal area values were between 10 and $20 \mathrm{~m}^{2} /$ ha. Distribution of trees by diameter class (Figure 3 ) demonstrate there is a

Table 1. List of family and species in alphabetical order and phytosociological descriptors estimation for the sampled tree comunities on 75 ha of dense "terra firme" rainforest located in Belo Monte Hydroelectric Plant's area of influence, situated in Pará, Brazil.

\begin{tabular}{|c|c|c|c|c|c|c|c|c|c|c|}
\hline FAMILY/SPECIES & AUTOR & N.ind & AD & RD & AF & RF & ADo & RDo & $\mathrm{CV}$ & IV \\
\hline \multicolumn{11}{|l|}{ ACHARIACEAE } \\
\hline Lindackeria paludosa & $\begin{array}{l}\text { (Benth) } \\
\text { Gilg }\end{array}$ & 86 & 1.15 & 0.317 & 0.301 & 0.509 & 1.406 & 0.129 & 0.223 & 0.318 \\
\hline Mayna odorata & Aubl & 6 & 0.08 & 0.022 & 0.014 & 0.023 & 0.126 & 0.012 & 0.017 & 0.019 \\
\hline \multicolumn{11}{|l|}{ ANACARDIACEAE } \\
\hline Anacardium giganteum & $\begin{array}{l}\text { W } \\
\text { Hancock } \\
\text { ex Engl }\end{array}$ & 12 & 0.16 & 0.044 & 0.096 & 0.162 & 1.364 & 0.125 & 0.085 & 0.110 \\
\hline Anacardium occidentale & $\mathrm{L}$ & 1 & 0.01 & 0.004 & 0.014 & 0.023 & 0.078 & 0.007 & 0.005 & 0.011 \\
\hline $\begin{array}{l}\text { Anacardium } \\
\text { spruceanum }\end{array}$ & $\begin{array}{l}\text { Benth ex } \\
\text { Engl }\end{array}$ & 5 & 0.07 & 0.018 & 0.041 & 0.069 & 0.354 & 0.032 & 0.025 & 0.040 \\
\hline Astronium gracile & Jacq & 17 & 0.23 & 0.063 & 0.110 & 0.185 & 0.981 & 0.090 & 0.076 & 0.113 \\
\hline Astronium lecointei & Ducke & 71 & 0.95 & 0.262 & 0.342 & 0.579 & 5.363 & 0.492 & 0.377 & 0.444 \\
\hline Mangifera indica & $\mathrm{L}$ & 1 & 0.01 & 0.004 & 0.014 & 0.023 & 0.117 & 0.011 & 0.007 & 0.013 \\
\hline Spondias mombin & $\mathrm{L}$ & 171 & 2.28 & 0.630 & 0.219 & 0.370 & 9.057 & 0.830 & 0.730 & 0.610 \\
\hline Tapirira guianensis & Aubl & 67 & 0.89 & 0.247 & 0.274 & 0.463 & 2.667 & 0.244 & 0.246 & 0.318 \\
\hline Thyrsodium spruceanum & Benth & 151 & 2.01 & 0.557 & 0.466 & 0.787 & 4.562 & 0.418 & 0.487 & 0.587 \\
\hline \multicolumn{11}{|c|}{ ANNONACEAE } \\
\hline Anaxagorea prinoides & $\begin{array}{l}\text { (Dunal) } \\
\text { A.DC }\end{array}$ & 6 & 0.08 & 0.022 & 0.027 & 0.046 & 0.090 & 0.008 & 0.015 & 0.026 \\
\hline Annona edulis & $\begin{array}{l}\text { (Triana \& } \\
\text { Planch) H } \\
\text { Rainer }\end{array}$ & 9 & 0.12 & 0.033 & 0.055 & 0.093 & 0.234 & 0.021 & 0.027 & 0.049 \\
\hline Annona exsucca & $\mathrm{DC}$ & 131 & 1.75 & 0.483 & 0.329 & 0.555 & 2.115 & 0.194 & 0.338 & 0.411 \\
\hline Annona hypoglauca & Mart & 4 & 0.05 & 0.015 & 0.014 & 0.023 & 0.043 & 0.004 & 0.009 & 0.014 \\
\hline Annona montana & Macfad & 6 & 0.08 & 0.022 & 0.027 & 0.046 & 0.054 & 0.005 & 0.014 & 0.024 \\
\hline Annona mucosa & Jacq & 4 & 0.05 & 0.015 & 0.027 & 0.046 & 0.047 & 0.004 & 0.010 & 0.022 \\
\hline Cardiopetalum sp & & 6 & 0.08 & 0.022 & 0.027 & 0.046 & 0.072 & 0.007 & 0.014 & 0.025 \\
\hline Duguetia echinophora & R E Fr & 12 & 0.16 & 0.044 & 0.027 & 0.046 & 0.266 & 0.024 & 0.034 & 0.038 \\
\hline Duguetia flagellaris & Huber & 2 & 0.03 & 0.007 & 0.014 & 0.023 & 0.022 & 0.002 & 0.005 & 0.011 \\
\hline Duguetia riparia & Huber & 4 & 0.05 & 0.015 & 0.027 & 0.046 & 0.198 & 0.018 & 0.016 & 0.026 \\
\hline Fusaea longifolia & (Aubl) Saff & 74 & 0.99 & 0.273 & 0.178 & 0.301 & 2.199 & 0.202 & 0.237 & 0.258 \\
\hline Guatteria poeppigiana & Mart & 146 & 1.95 & 0.538 & 0.438 & 0.741 & 4.171 & 0.382 & 0.460 & 0.554 \\
\hline $\begin{array}{l}\text { Guatteria } \\
\text { schomburgkiana }\end{array}$ & Mart & 24 & 0.32 & 0.088 & 0.041 & 0.069 & 0.526 & 0.048 & 0.068 & 0.069 \\
\hline Guatteria sp 2 & & 21 & 0.28 & 0.077 & 0.041 & 0.069 & 0.434 & 0.040 & 0.059 & 0.062 \\
\hline Oxandra euneura & Diels & 232 & 3.09 & 0.855 & 0.288 & 0.486 & 3.751 & 0.344 & 0.600 & 0.562 \\
\hline
\end{tabular}

$\mathrm{N}$. ind $=$ number of individuals, $\mathrm{AD}=$ absolute density, $\mathrm{RD}=$ relative density, $\mathrm{AF}=$ absolute frequency, $\mathrm{RF}=$ relative frequency,

$\mathrm{ADo}=$ absolute dominance, $\mathrm{RDo}=$ relative dominance, $\mathrm{CV}=$ coverage value and $\mathrm{IV}=$ importance value. 
Table 1. Continued...

\begin{tabular}{|c|c|c|c|c|c|c|c|c|c|c|}
\hline FAMILY/SPECIES & AUTOR & N.ind & AD & RD & AF & RF & ADo & RDo & $\mathrm{CV}$ & IV \\
\hline Unonopsis guatterioides & $\begin{array}{l}(\mathrm{A} \mathrm{DC}) \mathrm{R} \\
\mathrm{E} \text { Fr }\end{array}$ & 16 & 0.21 & 0.059 & 0.068 & 0.116 & 0.205 & 0.019 & 0.039 & 0.064 \\
\hline Xylopia amazonica & R E Fr & 10 & 0.13 & 0.037 & 0.068 & 0.116 & 0.219 & 0.020 & 0.028 & 0.058 \\
\hline Xylopia aromatica & (Lam) Mart & 33 & 0.44 & 0.122 & 0.137 & 0.231 & 0.816 & 0.075 & 0.098 & 0.143 \\
\hline Xylopia nitida & Dunal & 55 & 0.73 & 0.203 & 0.260 & 0.440 & 2.179 & 0.200 & 0.201 & 0.281 \\
\hline Xylopia sp 1 & & 2 & 0.03 & 0.007 & 0.014 & 0.023 & 0.022 & 0.002 & 0.005 & 0.011 \\
\hline \multicolumn{11}{|l|}{ APOCYNACEAE } \\
\hline $\begin{array}{l}\text { Aspidosperma } \\
\text { desmanthum }\end{array}$ & $\begin{array}{l}\text { Benth ex } \\
\text { Müll Arg }\end{array}$ & 86 & 1.15 & 0.317 & 0.384 & 0.648 & 3.706 & 0.340 & 0.328 & 0.435 \\
\hline Aspidosperma excelsum & Benth & 58 & 0.77 & 0.214 & 0.329 & 0.555 & 5.844 & 0.536 & 0.375 & 0.435 \\
\hline $\begin{array}{l}\text { Aspidosperma } \\
\text { sandwithianum }\end{array}$ & Markgr & 2 & 0.03 & 0.007 & 0.014 & 0.023 & 0.024 & 0.002 & 0.005 & 0.011 \\
\hline Aspidosperma sp & & 11 & 0.15 & 0.041 & 0.068 & 0.116 & 0.477 & 0.044 & 0.042 & 0.067 \\
\hline $\begin{array}{l}\text { Geissospermum } \\
\text { sericeum }\end{array}$ & Allemao & 17 & 0.23 & 0.063 & 0.082 & 0.139 & 1.764 & 0.162 & 0.112 & 0.121 \\
\hline Geissospermum vellosii & $\begin{array}{l}\text { (Vell) } \\
\text { Miers }\end{array}$ & 25 & 0.33 & 0.092 & 0.137 & 0.231 & 0.816 & 0.075 & 0.083 & 0.133 \\
\hline Himatanthus sucuuba & $\begin{array}{l}\text { (Spruce ex } \\
\text { Müll Arg) } \\
\text { Woodson }\end{array}$ & 36 & 0.48 & 0.133 & 0.164 & 0.278 & 1.029 & 0.094 & 0.114 & 0.168 \\
\hline Lacmellea aculeata & $\begin{array}{l}\text { (Ducke) } \\
\text { Monach }\end{array}$ & 13 & 0.17 & 0.048 & 0.055 & 0.093 & 0.492 & 0.045 & 0.046 & 0.062 \\
\hline Lacmellea arborescens & $\begin{array}{l}\text { (Müll Arg) } \\
\text { Markgr }\end{array}$ & 50 & 0.67 & 0.184 & 0.151 & 0.255 & 0.692 & 0.063 & 0.124 & 0.167 \\
\hline $\begin{array}{l}\text { Parahancornia } \\
\text { fasciculata }\end{array}$ & $\begin{array}{l}\text { (Poir) } \\
\text { Benoist }\end{array}$ & 1 & 0.01 & 0.004 & 0.014 & 0.023 & 0.078 & 0.007 & 0.005 & 0.011 \\
\hline $\begin{array}{l}\text { Tabernaemontana } \\
\text { siphilitica }\end{array}$ & $\begin{array}{l}\text { (L.f.) } \\
\text { Leeuwenb. }\end{array}$ & 6 & 0.08 & 0.022 & 0.027 & 0.046 & 0.049 & 0.004 & 0.013 & 0.024 \\
\hline \multicolumn{11}{|l|}{ ARALIACEAE } \\
\hline Schefflera morototoni & $\begin{array}{l}\text { (Aubl) } \\
\text { Maguire, } \\
\text { Steyerm \& } \\
\text { Frodin }\end{array}$ & 131 & 1.75 & 0.483 & 0.411 & 0.694 & 7.046 & 0.646 & 0.564 & 0.608 \\
\hline \multicolumn{11}{|l|}{ BIGNONIACEAE } \\
\hline $\begin{array}{l}\text { Handroanthus } \\
\text { impetiginosus }\end{array}$ & $\begin{array}{l}\text { (Mart } \\
\text { ex DC) } \\
\text { Mattos }\end{array}$ & 2 & 0.03 & 0.007 & 0.014 & 0.023 & 0.040 & 0.004 & 0.006 & 0.011 \\
\hline $\begin{array}{l}\text { Handroanthus } \\
\text { ochraceus }\end{array}$ & $\begin{array}{l}\text { (Cham) } \\
\text { Mattos }\end{array}$ & 21 & 0.28 & 0.077 & 0.110 & 0.185 & 2.111 & 0.194 & 0.135 & 0.152 \\
\hline $\begin{array}{l}\text { Handroanthus } \\
\text { serratifolius }\end{array}$ & $\begin{array}{l}\text { (A H } \\
\text { Gentry) S } \\
\text { Grose }\end{array}$ & 30 & 0.40 & 0.111 & 0.123 & 0.208 & 1.047 & 0.096 & 0.103 & 0.138 \\
\hline Jacaranda copaia & $\begin{array}{l}\text { (Aubl) D } \\
\text { Don }\end{array}$ & 461 & 6.15 & 1.699 & 0.493 & 0.833 & 17.518 & 1.606 & 1.653 & 1.379 \\
\hline \multicolumn{11}{|l|}{ BIXACEAE } \\
\hline $\begin{array}{l}\text { Cochlospermum } \\
\text { orinocense }\end{array}$ & $\begin{array}{l}\text { (Kunth) } \\
\text { Steud }\end{array}$ & 155 & 2.07 & 0.571 & 0.110 & 0.185 & 3.544 & 0.325 & 0.448 & 0.360 \\
\hline \multicolumn{11}{|l|}{ BORAGINACEAE } \\
\hline Cordia exaltata & Lam & 248 & 3.31 & 0.914 & 0.548 & 0.926 & 5.540 & 0.508 & 0.711 & 0.783 \\
\hline Cordia goeldiana & Huber & 62 & 0.83 & 0.229 & 0.041 & 0.069 & 1.786 & 0.164 & 0.196 & 0.154 \\
\hline Cordia nodosa & Lam & 24 & 0.32 & 0.088 & 0.137 & 0.231 & 0.288 & 0.026 & 0.057 & 0.115 \\
\hline Cordia scabrifolia & A DC & 32 & 0.43 & 0.118 & 0.068 & 0.116 & 0.414 & 0.038 & 0.078 & 0.091 \\
\hline Cordia sellowiana & Cham & 24 & 0.32 & 0.088 & 0.096 & 0.162 & 0.430 & 0.039 & 0.064 & 0.097 \\
\hline
\end{tabular}


Table 1. Continued...

\begin{tabular}{|c|c|c|c|c|c|c|c|c|c|c|}
\hline FAMILY/SPECIES & AUTOR & N.ind & AD & RD & AF & RF & ADo & RDo & $\mathbf{C V}$ & IV \\
\hline Cordia tetrandra & Aubl & 29 & 0.39 & 0.107 & 0.027 & 0.046 & 2.242 & 0.206 & 0.156 & 0.120 \\
\hline \multicolumn{11}{|l|}{ BURSERACEAE } \\
\hline $\begin{array}{l}\text { Crepidospermum } \\
\text { goudotianum }\end{array}$ & $\begin{array}{l}\text { (Tul) } \\
\text { Triana \& } \\
\text { Planch }\end{array}$ & 76 & 1.01 & 0.280 & 0.247 & 0.417 & 2.938 & 0.269 & 0.275 & 0.322 \\
\hline Protium apiculatum & Swart & 162 & 2.16 & 0.597 & 0.288 & 0.486 & 3.686 & 0.338 & 0.468 & 0.474 \\
\hline Protium heptaphyllum & $\begin{array}{l}\text { (Aubl) } \\
\text { Marchand }\end{array}$ & 2 & 0.03 & 0.007 & 0.014 & 0.023 & 0.138 & 0.013 & 0.010 & 0.014 \\
\hline Protium pallidum & Cuatrec & 17 & 0.23 & 0.063 & 0.096 & 0.162 & 0.461 & 0.042 & 0.052 & 0.089 \\
\hline Protium paniculatum & Engl & 2 & 0.03 & 0.007 & 0.014 & 0.023 & 0.016 & 0.001 & 0.004 & 0.011 \\
\hline Protium robustum & $\begin{array}{l}\text { (Swart) D } \\
\text { M Porter }\end{array}$ & 115 & 1.53 & 0.424 & 0.342 & 0.579 & 2.215 & 0.203 & 0.314 & 0.402 \\
\hline Protium subserratum & (Engl) Engl & 77 & 1.03 & 0.284 & 0.329 & 0.555 & 3.681 & 0.337 & 0.311 & 0.392 \\
\hline Protium tenuifolium & (Engl) Engl & 110 & 1.47 & 0.406 & 0.219 & 0.370 & 2.814 & 0.258 & 0.332 & 0.345 \\
\hline Protium trifoliatum & Engl & 7 & 0.09 & 0.026 & 0.027 & 0.046 & 0.218 & 0.020 & 0.023 & 0.031 \\
\hline Tetragastris altissima & $\begin{array}{l}\text { (Aubl) } \\
\text { Swart }\end{array}$ & 130 & 1.73 & 0.479 & 0.329 & 0.555 & 4.511 & 0.414 & 0.446 & 0.483 \\
\hline Tetragastris panamensis & $\begin{array}{l}\text { (Engl) } \\
\text { Kuntze }\end{array}$ & 355 & 4.73 & 1.309 & 0.356 & 0.602 & 15.721 & 1.441 & 1.375 & 1.117 \\
\hline Trattinnickia lawrancei & $\begin{array}{l}\text { Standl ex } \\
\text { Swart }\end{array}$ & 5 & 0.07 & 0.018 & 0.041 & 0.069 & 0.191 & 0.017 & 0.018 & 0.035 \\
\hline Trattinnickia rhoifolia & Willd & 31 & 0.41 & 0.114 & 0.123 & 0.208 & 1.355 & 0.124 & 0.119 & 0.149 \\
\hline \multicolumn{11}{|l|}{ CALOPHYLLACEAE } \\
\hline Caraipa densifolia & Mart & 3 & 0.04 & 0.011 & 0.014 & 0.023 & 0.123 & 0.011 & 0.011 & 0.015 \\
\hline \multicolumn{11}{|l|}{ CANNABACEAE } \\
\hline Trema micrantha & (L) Blume & 12 & 0.16 & 0.044 & 0.027 & 0.046 & 0.232 & 0.021 & 0.033 & 0.037 \\
\hline \multicolumn{11}{|l|}{ CAPPARACEAE } \\
\hline Capparis amazonica & (L) J Presl & 14 & 0.19 & 0.052 & 0.041 & 0.069 & 0.193 & 0.018 & 0.035 & 0.046 \\
\hline \multicolumn{11}{|l|}{ CARICACEAE } \\
\hline Jacaratia spinosa & $\begin{array}{l}\text { (Aubl) A } \\
\text { DC }\end{array}$ & 329 & 4.39 & 1.213 & 0.479 & 0.810 & 16.141 & 1.480 & 1.346 & 1.167 \\
\hline \multicolumn{11}{|l|}{ CARYOCARACEAE } \\
\hline Caryocar glabrum & (Aubl) Pers & 4 & 0.05 & 0.015 & 0.027 & 0.046 & 0.088 & 0.008 & 0.011 & 0.023 \\
\hline Caryocar villosum & (Aubl) Pers & 2 & 0.03 & 0.007 & 0.014 & 0.023 & 0.019 & 0.002 & 0.005 & 0.011 \\
\hline \multicolumn{11}{|l|}{ CELASTRACEAE } \\
\hline Maytenus myrsinoides & Reissek & 2 & 0.03 & 0.007 & 0.014 & 0.023 & 0.094 & 0.009 & 0.008 & 0.013 \\
\hline Maytenus sp 2 & & 1 & 0.01 & 0.004 & 0.014 & 0.023 & 0.089 & 0.008 & 0.006 & 0.012 \\
\hline \multicolumn{11}{|l|}{ CHRYSOBALANACEAE } \\
\hline Couepia guianensis & Aubl & 19 & 0.25 & 0.070 & 0.027 & 0.046 & 0.560 & 0.051 & 0.061 & 0.056 \\
\hline Hirtella bicornis & $\begin{array}{l}\text { Mart \& } \\
\text { Zucc }\end{array}$ & 2 & 0.03 & 0.007 & 0.014 & 0.023 & 0.017 & 0.002 & 0.004 & 0.011 \\
\hline Hirtella eriandra & Benth & 102 & 1.36 & 0.376 & 0.219 & 0.370 & 2.851 & 0.261 & 0.319 & 0.336 \\
\hline Hirtella hispidula & Miq & 8 & 0.11 & 0.029 & 0.027 & 0.046 & 0.144 & 0.013 & 0.021 & 0.030 \\
\hline Licania apetala & $\begin{array}{l}\text { (E Mey) } \\
\text { Fritsch }\end{array}$ & 4 & 0.05 & 0.015 & 0.027 & 0.046 & 0.074 & 0.007 & 0.011 & 0.023 \\
\hline Licania guianensis & $\begin{array}{l}\text { (Aubl) } \\
\text { Griseb }\end{array}$ & 15 & 0.20 & 0.055 & 0.110 & 0.185 & 0.922 & 0.084 & 0.070 & 0.108 \\
\hline Licania heteromorpha & Benth & 113 & 1.51 & 0.417 & 0.384 & 0.648 & 3.275 & 0.300 & 0.358 & 0.455 \\
\hline Licania longistyla & $\begin{array}{l}(\text { Hook f) } \\
\text { Fritsch }\end{array}$ & 22 & 0.29 & 0.081 & 0.055 & 0.093 & 0.583 & 0.053 & 0.067 & 0.076 \\
\hline Licania membranacea & $\begin{array}{l}\text { Sagot ex } \\
\text { Laness }\end{array}$ & 9 & 0.12 & 0.033 & 0.068 & 0.116 & 0.269 & 0.025 & 0.029 & 0.058 \\
\hline
\end{tabular}

$\mathrm{N}$.ind $=$ number of individuals, $\mathrm{AD}=$ absolute density, $\mathrm{RD}=$ relative density, $\mathrm{AF}=$ absolute frequency, $\mathrm{RF}=$ relative frequency, $\mathrm{ADo}=$ absolute dominance, $\mathrm{RDo}=$ relative dominance, $\mathrm{CV}=$ coverage value and $\mathrm{IV}=$ importance value. 
Table 1. Continued...

\begin{tabular}{|c|c|c|c|c|c|c|c|c|c|c|}
\hline FAMILY/SPECIES & AUTOR & N.ind & AD & RD & $\mathbf{A F}$ & RF & ADo & RDo & $\mathbf{C V}$ & IV \\
\hline Licania octandra & $\begin{array}{l}\text { (Hoffmanns } \\
\text { ex Roem } \\
\& \text { Schult) } \\
\text { Kuntze }\end{array}$ & 19 & 0.25 & 0.070 & 0.068 & 0.116 & 0.590 & 0.054 & 0.062 & 0.080 \\
\hline Licania sclerophylla & $\begin{array}{l}\text { (Hook f) } \\
\text { Fritsch }\end{array}$ & 2 & 0.03 & 0.007 & 0.014 & 0.023 & 0.045 & 0.004 & 0.006 & 0.012 \\
\hline Licania unguiculata & Prance & 3 & 0.04 & 0.011 & 0.027 & 0.046 & 0.223 & 0.020 & 0.016 & 0.026 \\
\hline Parinari excelsa & Sabine & 54 & 0.72 & 0.199 & 0.192 & 0.324 & 3.086 & 0.283 & 0.241 & 0.269 \\
\hline \multicolumn{11}{|l|}{ CLUSIACEAE } \\
\hline Carapa guianensis & Aubl & 157 & 2.09 & 0.579 & 0.260 & 0.440 & 10.377 & 0.951 & 0.765 & 0.657 \\
\hline Garcinia gardneriana & $\begin{array}{l}\text { (Planch } \\
\text { \& Triana) } \\
\text { Zappi }\end{array}$ & 12 & 0.16 & 0.044 & 0.055 & 0.093 & 0.120 & 0.011 & 0.028 & 0.049 \\
\hline Garcinia macrophylla & Mart & 45 & 0.60 & 0.166 & 0.164 & 0.278 & 0.926 & 0.085 & 0.125 & 0.176 \\
\hline Moronobea candida & Ducke & 2 & 0.03 & 0.007 & 0.014 & 0.023 & 0.035 & 0.003 & 0.005 & 0.011 \\
\hline Symphonia globulifera & Lf & 5 & 0.07 & 0.018 & 0.041 & 0.069 & 0.259 & 0.024 & 0.021 & 0.037 \\
\hline Tovomita choisyana & $\begin{array}{l}\text { Planch \& } \\
\text { Triana }\end{array}$ & 1 & 0.01 & 0.004 & 0.014 & 0.023 & 0.080 & 0.007 & 0.006 & 0.011 \\
\hline \multicolumn{11}{|l|}{ COMBRETACEAE } \\
\hline Buchenavia grandis & Ducke & 5 & 0.07 & 0.018 & 0.041 & 0.069 & 2.428 & 0.223 & 0.121 & 0.103 \\
\hline Buchenavia oxycarpa & $\begin{array}{l}\text { (Mart) } \\
\text { Eichler }\end{array}$ & 12 & 0.16 & 0.044 & 0.014 & 0.023 & 0.324 & 0.030 & 0.037 & 0.032 \\
\hline Terminalia amazonia & $\begin{array}{l}\text { (J F Gmel) } \\
\text { Exell }\end{array}$ & 29 & 0.39 & 0.107 & 0.137 & 0.231 & 2.668 & 0.245 & 0.176 & 0.194 \\
\hline Terminalia argentea & Mart & 20 & 0.27 & 0.074 & 0.110 & 0.185 & 1.722 & 0.158 & 0.116 & 0.139 \\
\hline Terminalia dichotoma & E Mey & 5 & 0.07 & 0.018 & 0.041 & 0.069 & 0.844 & 0.077 & 0.048 & 0.055 \\
\hline \multicolumn{11}{|l|}{ CONNARACEAE } \\
\hline Connarus erianthus & $\begin{array}{l}\text { Benth ex } \\
\text { Baker }\end{array}$ & 2 & 0.03 & 0.007 & 0.014 & 0.023 & 0.016 & 0.001 & 0.004 & 0.011 \\
\hline \multicolumn{11}{|l|}{ EBENACEAE } \\
\hline Diospyros artanthifolia & Mart & 12 & 0.16 & 0.044 & 0.055 & 0.093 & 0.175 & 0.016 & 0.030 & 0.051 \\
\hline Diospyros capreifolia & $\begin{array}{l}\text { Mart ex } \\
\text { Hiern }\end{array}$ & 4 & 0.05 & 0.015 & 0.014 & 0.023 & 0.073 & 0.007 & 0.011 & 0.015 \\
\hline Diospyros guianensis & $\begin{array}{l}\text { (Aubl) } \\
\text { Gürke }\end{array}$ & 9 & 0.12 & 0.033 & 0.055 & 0.093 & 5.478 & 0.502 & 0.268 & 0.209 \\
\hline Diospyros vestita & Benoist & 138 & 1.84 & 0.509 & 0.425 & 0.717 & 4.869 & 0.446 & 0.478 & 0.558 \\
\hline \multicolumn{11}{|l|}{ ELAEOCARPACEAE } \\
\hline Sloanea eichleri & K Schum & 5 & 0.07 & 0.018 & 0.027 & 0.046 & 0.252 & 0.023 & 0.021 & 0.029 \\
\hline Sloanea garckeana & K Schum & 2 & 0.03 & 0.007 & 0.014 & 0.023 & 0.058 & 0.005 & 0.006 & 0.012 \\
\hline Sloanea grandiflora & $\mathrm{Sm}$ & 63 & 0.84 & 0.232 & 0.192 & 0.324 & 1.429 & 0.131 & 0.182 & 0.229 \\
\hline Sloanea guianensis & $\begin{array}{l}\text { (Aubl) } \\
\text { Benth }\end{array}$ & 2 & 0.03 & 0.007 & 0.014 & 0.023 & 0.075 & 0.007 & 0.007 & 0.012 \\
\hline \multicolumn{11}{|c|}{ ERYTHROXYLACEAE } \\
\hline Erythroxylum amplum & Benth & 4 & 0.05 & 0.015 & 0.014 & 0.023 & 0.051 & 0.005 & 0.010 & 0.014 \\
\hline \multicolumn{11}{|l|}{ EUPHORBIACEAE } \\
\hline $\begin{array}{l}\text { Actinostemon } \\
\text { amazonicus }\end{array}$ & $\begin{array}{l}\text { Pax \& K. } \\
\text { Hoffm. }\end{array}$ & 10 & 0.13 & 0.037 & 0.027 & 0.046 & 0.136 & 0.012 & 0.025 & 0.032 \\
\hline Alchornea schomburgkii & Poepp & 2 & 0.03 & 0.007 & 0.014 & 0.023 & 0.026 & 0.002 & 0.005 & 0.011 \\
\hline Croton glandulosus & $\mathrm{L}$ & 20 & 0.27 & 0.074 & 0.041 & 0.069 & 0.308 & 0.028 & 0.051 & 0.057 \\
\hline Croton $s p$ & & 10 & 0.13 & 0.037 & 0.027 & 0.046 & 0.136 & 0.012 & 0.025 & 0.032 \\
\hline $\begin{array}{l}\text { Dodecastigma } \\
\text { integrifolium }\end{array}$ & $\begin{array}{l}\text { (Lanj) } \\
\text { Lanj \& } \\
\text { Sandwith }\end{array}$ & 14 & 0.19 & 0.052 & 0.055 & 0.093 & 0.150 & 0.014 & 0.033 & 0.053 \\
\hline
\end{tabular}

$\mathrm{N}$.ind = number of individuals, $\mathrm{AD}=$ absolute density, $\mathrm{RD}=$ relative density, $\mathrm{AF}=$ absolute frequency, $\mathrm{RF}=$ relative frequency, $\mathrm{ADo}=$ absolute dominance, $\mathrm{RDo}=$ relative dominance, $\mathrm{CV}=$ coverage value and $\mathrm{IV}=$ importance value. 
Table 1. Continued...

\begin{tabular}{|c|c|c|c|c|c|c|c|c|c|c|}
\hline FAMILY/SPECIES & AUTOR & N.ind & AD & RD & $\mathbf{A F}$ & RF & ADo & RDo & $\mathbf{C V}$ & IV \\
\hline $\begin{array}{l}\text { Glycydendron } \\
\text { amazonicum }\end{array}$ & Ducke & 2 & 0.03 & 0.007 & 0.014 & 0.023 & 0.090 & 0.008 & 0.008 & 0.013 \\
\hline Hevea brasiliensis & $\begin{array}{l}\text { (Willd ex A } \\
\text { Juss) Müll } \\
\text { Arg }\end{array}$ & 53 & 0.71 & 0.195 & 0.096 & 0.162 & 5.165 & 0.473 & 0.334 & 0.277 \\
\hline Mabea nitida & $\begin{array}{l}\text { Spruce ex } \\
\text { benth. }\end{array}$ & 527 & 7.03 & 1.943 & 0.137 & 0.231 & 15.796 & 1.448 & 1.695 & 1.207 \\
\hline Mabea paniculata & $\begin{array}{l}\text { Spruce ex } \\
\text { Benth. }\end{array}$ & 25 & 0.33 & 0.092 & 0.014 & 0.023 & 0.956 & 0.088 & 0.090 & 0.068 \\
\hline Maprounea guianensis & $\begin{array}{l}\text { (Miq) T D } \\
\text { Penn }\end{array}$ & 12 & 0.16 & 0.044 & 0.055 & 0.093 & 0.182 & 0.017 & 0.030 & 0.051 \\
\hline Sagotia racemosa & Baill & 2 & 0.03 & 0.007 & 0.014 & 0.023 & 0.030 & 0.003 & 0.005 & 0.011 \\
\hline Sapium glandulosum & $\begin{array}{l}\text { (L) } \\
\text { Morong }\end{array}$ & 6 & 0.08 & 0.022 & 0.027 & 0.046 & 0.168 & 0.015 & 0.019 & 0.028 \\
\hline Sapium lanceolatum & $\begin{array}{l}\text { (L) } \\
\text { Morong }\end{array}$ & 175 & 2.33 & 0.645 & 0.466 & 0.787 & 7.491 & 0.687 & 0.666 & 0.706 \\
\hline Sapium marmieri & Huber & 164 & 2.19 & 0.605 & 0.342 & 0.579 & 4.496 & 0.412 & 0.508 & 0.532 \\
\hline \multicolumn{11}{|l|}{ FABACEAE } \\
\hline Abarema jupunba & $\begin{array}{l}\text { (Willd) } \\
\text { Britton \& } \\
\text { Killip }\end{array}$ & 99 & 1.32 & 0.365 & 0.274 & 0.463 & 3.674 & 0.337 & 0.351 & 0.388 \\
\hline Abarema mataybifolia & $\begin{array}{l}\text { (Sandwith) } \\
\text { Barneby \& } \\
\text { J W Grime }\end{array}$ & 3 & 0.04 & 0.011 & 0.027 & 0.046 & 0.149 & 0.014 & 0.012 & 0.024 \\
\hline Albizia niopoides & $\begin{array}{l}\text { (Spruce } \\
\text { ex Benth) } \\
\text { Burkart }\end{array}$ & 3 & 0.04 & 0.011 & 0.027 & 0.046 & 0.123 & 0.011 & 0.011 & 0.023 \\
\hline Albizia pedicellaris & $\begin{array}{l}\text { (DC) L } \\
\text { Rico }\end{array}$ & 31 & 0.41 & 0.114 & 0.137 & 0.231 & 3.212 & 0.294 & 0.204 & 0.213 \\
\hline Alexa grandiflora & Ducke & 890 & 11.87 & 3.281 & 0.685 & 1.157 & 96.202 & 8.819 & 6.050 & 4.419 \\
\hline Amphiodon effusus & Huber & 44 & 0.59 & 0.162 & 0.082 & 0.139 & 0.601 & 0.055 & 0.109 & 0.119 \\
\hline Andira surinamensis & $\begin{array}{l}\text { (Bondt) } \\
\text { Splitg ex } \\
\text { Amshoff }\end{array}$ & 2 & 0.03 & 0.007 & 0.014 & 0.023 & 0.028 & 0.003 & 0.005 & 0.011 \\
\hline Apuleia leiocarpa & $\begin{array}{l}\text { (Vogel) J F } \\
\text { Macbr }\end{array}$ & 108 & 1.44 & 0.398 & 0.397 & 0.671 & 8.428 & 0.773 & 0.585 & 0.614 \\
\hline Bauhinia acreana & Harms & 101 & 1.35 & 0.372 & 0.233 & 0.393 & 2.235 & 0.205 & 0.289 & 0.324 \\
\hline Bauhinia longicuspis & Benth & 59 & 0.79 & 0.218 & 0.082 & 0.139 & 1.570 & 0.144 & 0.181 & 0.167 \\
\hline Campsiandra laurifolia & Benth & 2 & 0.03 & 0.007 & 0.014 & 0.023 & 0.112 & 0.010 & 0.009 & 0.014 \\
\hline Cassia fastuosa & $\begin{array}{l}\text { Willd ex } \\
\text { Benth }\end{array}$ & 29 & 0.39 & 0.107 & 0.137 & 0.231 & 1.334 & 0.122 & 0.115 & 0.154 \\
\hline Cenostigma tocantinum & Ducke & 835 & 11.13 & 3.078 & 0.260 & 0.440 & 43.725 & 4.008 & 3.543 & 2.509 \\
\hline Chamaecrista bahiae & $\begin{array}{l}\text { (H S Irwin) } \\
\text { H S Irwin } \\
\text { \& Barneby }\end{array}$ & 19 & 0.25 & 0.070 & 0.027 & 0.046 & 0.408 & 0.037 & 0.054 & 0.051 \\
\hline $\begin{array}{l}\text { Chamaecrista } \\
\text { xinguensis }\end{array}$ & $\begin{array}{l}\text { (Ducke) H } \\
\text { S Irwin \& } \\
\text { Barneby }\end{array}$ & 172 & 2.29 & 0.634 & 0.329 & 0.555 & 5.305 & 0.486 & 0.560 & 0.559 \\
\hline Copaifera martii & Hayne & 8 & 0.11 & 0.029 & 0.068 & 0.116 & 0.363 & 0.033 & 0.031 & 0.059 \\
\hline Copaifera multijuga & Hayne & 1 & 0.01 & 0.004 & 0.014 & 0.023 & 0.269 & 0.025 & 0.014 & 0.017 \\
\hline Crudia oblonga & Benth & 31 & 0.41 & 0.114 & 0.027 & 0.046 & 1.464 & 0.134 & 0.124 & 0.098 \\
\hline Crudia tomentosa & $\begin{array}{l}\text { (Aubl) J F } \\
\text { Macbr }\end{array}$ & 10 & 0.13 & 0.037 & 0.027 & 0.046 & 0.298 & 0.027 & 0.032 & 0.037 \\
\hline Cynometra marginata & Benth & 46 & 0.61 & 0.170 & 0.055 & 0.093 & 3.952 & 0.362 & 0.266 & 0.208 \\
\hline
\end{tabular}

$\mathrm{N}$. ind $=$ number of individuals, $\mathrm{AD}=$ absolute density, $\mathrm{RD}=$ relative density, $\mathrm{AF}=$ absolute frequency, $\mathrm{RF}=$ relative frequency, $\mathrm{ADo}=$ absolute dominance, $\mathrm{RDo}=$ relative dominance, $\mathrm{CV}=$ coverage value and $\mathrm{IV}=$ importance value. 
Table 1. Continued...

\begin{tabular}{|c|c|c|c|c|c|c|c|c|c|c|}
\hline FAMILY/SPECIES & AUTOR & N.ind & AD & RD & $\mathbf{A F}$ & RF & ADo & RDo & $\mathbf{C V}$ & IV \\
\hline Dialium guianense & $\begin{array}{l}\text { (Aubl) } \\
\text { Sandwith }\end{array}$ & 172 & 2.29 & 0.634 & 0.493 & 0.833 & 4.863 & 0.446 & 0.540 & 0.638 \\
\hline Diplotropis purpurea & $\begin{array}{l}\text { (Rich) } \\
\text { Amshoff }\end{array}$ & 11 & 0.15 & 0.041 & 0.068 & 0.116 & 0.264 & 0.024 & 0.032 & 0.060 \\
\hline Dipteryx odorata & $\begin{array}{l}\text { (Aubl) } \\
\text { Willd }\end{array}$ & 16 & 0.21 & 0.059 & 0.110 & 0.185 & 0.976 & 0.089 & 0.074 & 0.111 \\
\hline Dussia discolor & $\begin{array}{l}\text { (Benth) } \\
\text { Amshoff }\end{array}$ & 3 & 0.04 & 0.011 & 0.027 & 0.046 & 0.200 & 0.018 & 0.015 & 0.025 \\
\hline Enterolobium maximum & Ducke & 12 & 0.16 & 0.044 & 0.110 & 0.185 & 1.584 & 0.145 & 0.095 & 0.125 \\
\hline $\begin{array}{l}\text { Enterolobium } \\
\text { schomburgkii }\end{array}$ & $\begin{array}{l}\text { (Benth) } \\
\text { Benth }\end{array}$ & 26 & 0.35 & 0.096 & 0.192 & 0.324 & 2.045 & 0.187 & 0.142 & 0.202 \\
\hline Erythrina fusca & Lour & 35 & 0.47 & 0.129 & 0.192 & 0.324 & 1.306 & 0.120 & 0.124 & 0.191 \\
\hline Hydrochorea corymbosa & $\begin{array}{l}\text { (Rich) } \\
\text { Barneby } \\
\text { \& J W } \\
\text { Grimes }\end{array}$ & 22 & 0.29 & 0.081 & 0.027 & 0.046 & 0.716 & 0.066 & 0.073 & 0.064 \\
\hline Hymenaea courbaril & $\mathrm{L}$ & 26 & 0.35 & 0.096 & 0.151 & 0.255 & 6.594 & 0.604 & 0.350 & 0.318 \\
\hline Hymenaea intermedia & Ducke & 22 & 0.29 & 0.081 & 0.096 & 0.162 & 1.180 & 0.108 & 0.095 & 0.117 \\
\hline Hymenaea oblongifolia & Huber & 17 & 0.23 & 0.063 & 0.055 & 0.093 & 0.649 & 0.059 & 0.061 & 0.072 \\
\hline Hymenaea parvifolia & Huber & 7 & 0.09 & 0.026 & 0.055 & 0.093 & 0.619 & 0.057 & 0.041 & 0.058 \\
\hline $\begin{array}{l}\text { Hymenolobium } \\
\text { petraeum }\end{array}$ & Ducke & 8 & 0.11 & 0.029 & 0.055 & 0.093 & 0.115 & 0.011 & 0.020 & 0.044 \\
\hline Inga alba & (Sw) Willd & 327 & 4.36 & 1.205 & 0.603 & 1.018 & 13.518 & 1.239 & 1.222 & 1.154 \\
\hline Inga auristellae & Harms & 6 & 0.08 & 0.022 & 0.041 & 0.069 & 0.079 & 0.007 & 0.015 & 0.033 \\
\hline Inga brachystachys & Ducke & 262 & 3.49 & 0.966 & 0.521 & 0.879 & 5.876 & 0.539 & 0.752 & 0.795 \\
\hline Inga capitata & Desv & 57 & 0.76 & 0.210 & 0.233 & 0.393 & 1.181 & 0.108 & 0.159 & 0.237 \\
\hline Inga cayennensis & $\begin{array}{l}\text { Sagot ex } \\
\text { Benth }\end{array}$ & 39 & 0.52 & 0.144 & 0.151 & 0.255 & 0.970 & 0.089 & 0.116 & 0.162 \\
\hline Inga edulis & Mart & 505 & 6.73 & 1.862 & 0.712 & 1.203 & 21.884 & 2.006 & 1.934 & 1.690 \\
\hline Inga gracilifolia & Ducke & 2 & 0.03 & 0.007 & 0.014 & 0.023 & 0.016 & 0.001 & 0.004 & 0.011 \\
\hline Inga grandiflora & Ducke & 10 & 0.13 & 0.037 & 0.041 & 0.069 & 0.154 & 0.014 & 0.026 & 0.040 \\
\hline Inga heterophylla & Willd & 115 & 1.53 & 0.424 & 0.397 & 0.671 & 2.713 & 0.249 & 0.336 & 0.448 \\
\hline Inga laurina & (Sw) Willd & 28 & 0.37 & 0.103 & 0.137 & 0.231 & 0.619 & 0.057 & 0.080 & 0.130 \\
\hline Inga nobilis & Willd & 16 & 0.21 & 0.059 & 0.055 & 0.093 & 0.191 & 0.018 & 0.038 & 0.056 \\
\hline Inga paraensis & Ducke & 248 & 3.31 & 0.914 & 0.479 & 0.810 & 7.737 & 0.709 & 0.812 & 0.811 \\
\hline Inga rubiginosa & (Rich) DC & 195 & 2.60 & 0.719 & 0.205 & 0.347 & 8.886 & 0.815 & 0.767 & 0.627 \\
\hline Inga sapindoides & Willd & 2 & 0.03 & 0.007 & 0.014 & 0.023 & 0.019 & 0.002 & 0.005 & 0.011 \\
\hline Inga sp 3 & & 2 & 0.03 & 0.007 & 0.014 & 0.023 & 0.031 & 0.003 & 0.005 & 0.011 \\
\hline Inga splendens & Willd & 16 & 0.21 & 0.059 & 0.082 & 0.139 & 0.255 & 0.023 & 0.041 & 0.074 \\
\hline Inga thibaudiana & $\mathrm{DC}$ & 166 & 2.21 & 0.612 & 0.329 & 0.555 & 3.061 & 0.281 & 0.446 & 0.483 \\
\hline Machaerium sp 1 & & 1 & 0.01 & 0.004 & 0.014 & 0.023 & 0.165 & 0.015 & 0.009 & 0.014 \\
\hline Machaerium sp 2 & & 4 & 0.05 & 0.015 & 0.014 & 0.023 & 0.077 & 0.007 & 0.011 & 0.015 \\
\hline $\begin{array}{l}\text { Macrolobium } \\
\text { acaciifolium }\end{array}$ & Benth & 4 & 0.05 & 0.015 & 0.014 & 0.023 & 0.047 & 0.004 & 0.010 & 0.014 \\
\hline Macrolobium bifolium & (Aubl) Pers & 4 & 0.05 & 0.015 & 0.027 & 0.046 & 0.152 & 0.014 & 0.014 & 0.025 \\
\hline Mora paraensis & $\begin{array}{l}\text { (Ducke) } \\
\text { Ducke }\end{array}$ & 12 & 0.16 & 0.044 & 0.014 & 0.023 & 1.487 & 0.136 & 0.090 & 0.068 \\
\hline Mucuna urens & (L) Medik & 4 & 0.05 & 0.015 & 0.027 & 0.046 & 0.098 & 0.009 & 0.012 & 0.023 \\
\hline Ormosia coutinhoi & Ducke & 2 & 0.03 & 0.007 & 0.014 & 0.023 & 0.094 & 0.009 & 0.008 & 0.013 \\
\hline Ormosia flava & $\begin{array}{l}\text { (Ducke) } \\
\text { Rudd }\end{array}$ & 4 & 0.05 & 0.015 & 0.041 & 0.069 & 0.652 & 0.060 & 0.037 & 0.048 \\
\hline Ormosia paraensis & Ducke & 6 & 0.08 & 0.022 & 0.041 & 0.069 & 0.062 & 0.006 & 0.014 & 0.032 \\
\hline
\end{tabular}


Table 1. Continued...

\begin{tabular}{|c|c|c|c|c|c|c|c|c|c|c|}
\hline FAMILY/SPECIES & AUTOR & N.ind & AD & RD & $\mathbf{A F}$ & RF & ADo & RDo & $\mathbf{C V}$ & IV \\
\hline Parkia multijuga & Benth & 40 & 0.53 & 0.147 & 0.219 & 0.370 & 6.884 & 0.631 & 0.389 & 0.383 \\
\hline Parkia pendula & $\begin{array}{l}\text { (Willd) } \\
\text { Benth ex } \\
\text { Walp }\end{array}$ & 12 & 0.16 & 0.044 & 0.110 & 0.185 & 3.865 & 0.354 & 0.199 & 0.195 \\
\hline Parkia ulei & $\begin{array}{l}\text { (Harms) } \\
\text { Kuhlm }\end{array}$ & 1 & 0.01 & 0.004 & 0.014 & 0.023 & 0.081 & 0.007 & 0.006 & 0.011 \\
\hline Parkia velutina & Benoist & 2 & 0.03 & 0.007 & 0.014 & 0.023 & 0.077 & 0.007 & 0.007 & 0.013 \\
\hline Platymiscium filipes & Benth & 13 & 0.17 & 0.048 & 0.096 & 0.162 & 0.862 & 0.079 & 0.063 & 0.096 \\
\hline $\begin{array}{l}\text { Pseudopiptadenia } \\
\text { psilostachya }\end{array}$ & $\begin{array}{l}\text { (DC) G P } \\
\text { Lewis \& M } \\
\text { P Lima }\end{array}$ & 40 & 0.53 & 0.147 & 0.164 & 0.278 & 3.796 & 0.348 & 0.248 & 0.258 \\
\hline $\begin{array}{l}\text { Pseudopiptadenia } \\
\text { suaveolens }\end{array}$ & $\begin{array}{l}\text { (Miq) J W } \\
\text { Grimes }\end{array}$ & 3 & 0.04 & 0.011 & 0.027 & 0.046 & 0.153 & 0.014 & 0.013 & 0.024 \\
\hline Pterocarpus amazonum & $\begin{array}{l}\text { L'Her ex } \\
\text { DC }\end{array}$ & 17 & 0.23 & 0.063 & 0.014 & 0.023 & 0.576 & 0.053 & 0.058 & 0.046 \\
\hline Pterocarpus officinalis & Jacq & 115 & 1.53 & 0.424 & 0.233 & 0.393 & 9.248 & 0.848 & 0.636 & 0.555 \\
\hline Pterocarpus rohrii & Vahl & 16 & 0.21 & 0.059 & 0.096 & 0.162 & 0.624 & 0.057 & 0.058 & 0.093 \\
\hline Samanea saman & (Jacq) Merr & 3 & 0.04 & 0.011 & 0.014 & 0.023 & 0.287 & 0.026 & 0.019 & 0.020 \\
\hline $\begin{array}{l}\text { Schizolobium parahyba } \\
\text { var Amazonicum }\end{array}$ & $\begin{array}{l}\text { (Huber ex } \\
\text { Ducke) } \\
\text { Barneby }\end{array}$ & 315 & 4.20 & 1.161 & 0.315 & 0.532 & 18.452 & 1.691 & 1.426 & 1.128 \\
\hline Senegalia polyphylla & $\begin{array}{l}\text { (DC) } \\
\text { Britton \& } \\
\text { Rose } \\
\end{array}$ & 155 & 2.07 & 0.571 & 0.247 & 0.417 & 4.442 & 0.407 & 0.489 & 0.465 \\
\hline Senna multijuga & $\begin{array}{l}\text { (Rich) H } \\
\text { S Irwin \& } \\
\text { Barneby } \\
\end{array}$ & 234 & 3.12 & 0.863 & 0.315 & 0.532 & 6.523 & 0.598 & 0.730 & 0.664 \\
\hline Senna silvestris & $\begin{array}{l}\text { (Vell) H } \\
\text { S Irwin \& } \\
\text { Barneby }\end{array}$ & 6 & 0.08 & 0.022 & 0.014 & 0.023 & 0.136 & 0.012 & 0.017 & 0.019 \\
\hline $\begin{array}{l}\text { Stryphnodendron } \\
\text { adstringens }\end{array}$ & $\begin{array}{l}\text { (Mart) } \\
\text { Coville }\end{array}$ & 45 & 0.60 & 0.166 & 0.137 & 0.231 & 0.877 & 0.080 & 0.123 & 0.159 \\
\hline $\begin{array}{l}\text { Stryphnodendron } \\
\text { guianense }\end{array}$ & $\begin{array}{l}\text { (Aubl) } \\
\text { Benth }\end{array}$ & 107 & 1.43 & 0.394 & 0.315 & 0.532 & 3.759 & 0.345 & 0.370 & 0.424 \\
\hline $\begin{array}{l}\text { Stryphnodendron } \\
\text { paniculatum }\end{array}$ & $\begin{array}{l}\text { Poepp \& } \\
\text { Endl }\end{array}$ & 49 & 0.65 & 0.181 & 0.233 & 0.393 & 4.591 & 0.421 & 0.301 & 0.332 \\
\hline $\begin{array}{l}\text { Stryphnodendron } \\
\text { pulcherrimum }\end{array}$ & $\begin{array}{l}\text { (Willd) } \\
\text { Hochr }\end{array}$ & 10 & 0.13 & 0.037 & 0.041 & 0.069 & 0.184 & 0.017 & 0.027 & 0.041 \\
\hline Swartzia arborescens & $\begin{array}{l}\text { (Aubl) } \\
\text { Pittier }\end{array}$ & 14 & 0.19 & 0.052 & 0.041 & 0.069 & 0.265 & 0.024 & 0.038 & 0.048 \\
\hline Swartzia flaemingii & Raddi & 42 & 0.56 & 0.155 & 0.178 & 0.301 & 0.745 & 0.068 & 0.112 & 0.175 \\
\hline Swartzia laurifolia & Benth & 85 & 1.13 & 0.313 & 0.425 & 0.717 & 2.063 & 0.189 & 0.251 & 0.407 \\
\hline Swartzia polyphylla & DC & 6 & 0.08 & 0.022 & 0.055 & 0.093 & 0.534 & 0.049 & 0.036 & 0.055 \\
\hline Swartzia sp & & 5 & 0.07 & 0.018 & 0.041 & 0.069 & 0.308 & 0.028 & 0.023 & 0.039 \\
\hline Tachigali chrysophylla & Poepp & 7 & 0.09 & 0.026 & 0.027 & 0.046 & 1.483 & 0.136 & 0.081 & 0.069 \\
\hline Tachigali myrmecophila & $\begin{array}{l}\text { (Ducke) } \\
\text { Ducke }\end{array}$ & 326 & 4.35 & 1.202 & 0.712 & 1.203 & 9.640 & 0.884 & 1.043 & 1.096 \\
\hline Tachigali paniculata & Aubl & 17 & 0.23 & 0.063 & 0.082 & 0.139 & 1.489 & 0.136 & 0.100 & 0.113 \\
\hline Vataieropsis $s p$ & & 19 & 0.25 & 0.070 & 0.055 & 0.093 & 0.818 & 0.075 & 0.073 & 0.079 \\
\hline Vatairea guianensis & Aubl & 30 & 0.40 & 0.111 & 0.041 & 0.069 & 1.732 & 0.159 & 0.135 & 0.113 \\
\hline Vouacapoua americana & Aubl & 651 & 8.68 & 2.400 & 0.438 & 0.741 & 39.868 & 3.655 & 3.027 & 2.265 \\
\hline Zollernia paraensis & Huber & 33 & 0.44 & 0.122 & 0.151 & 0.255 & 1.523 & 0.140 & 0.131 & 0.172 \\
\hline Zygia cauliflora & $\begin{array}{l}\text { (Willd) } \\
\text { Killip }\end{array}$ & 19 & 0.25 & 0.070 & 0.055 & 0.093 & 0.305 & 0.028 & 0.049 & 0.064 \\
\hline
\end{tabular}

$\mathrm{N}$.ind $=$ number of individuals, $\mathrm{AD}=$ absolute density, $\mathrm{RD}=$ relative density, $\mathrm{AF}=$ absolute frequency, $\mathrm{RF}=$ relative frequency, $\mathrm{ADo}=$ absolute dominance, $\mathrm{RDo}=$ relative dominance, $\mathrm{CV}=$ coverage value and $\mathrm{IV}=$ importance value. 
Table 1. Continued...

\begin{tabular}{|c|c|c|c|c|c|c|c|c|c|c|}
\hline FAMILY/SPECIES & AUTOR & N.ind & AD & RD & AF & RF & ADo & RDo & $\mathrm{CV}$ & IV \\
\hline Zygia inundata & $\begin{array}{l}\text { (Ducke) H } \\
\text { C Lima ex } \\
\text { Barneby \& } \\
\text { Grimes }\end{array}$ & 5 & 0.07 & 0.018 & 0.027 & 0.046 & 0.192 & 0.018 & 0.018 & 0.027 \\
\hline Zygia latifolia & $\begin{array}{l}\text { (L) Fawc } \\
\& \text { Rendle }\end{array}$ & 59 & 0.79 & 0.218 & 0.123 & 0.208 & 1.318 & 0.121 & 0.169 & 0.182 \\
\hline Zygia racemosa & $\begin{array}{l}\text { (Ducke) } \\
\text { Barneby } \\
\text { \& J W } \\
\text { Grimes }\end{array}$ & 16 & 0.21 & 0.059 & 0.041 & 0.069 & 0.413 & 0.038 & 0.048 & 0.055 \\
\hline \multicolumn{11}{|l|}{ GOUPIACEAE } \\
\hline Goupia glabra & Aubl & 1 & 0.01 & 0.004 & 0.014 & 0.023 & 0.179 & 0.016 & 0.010 & 0.014 \\
\hline \multicolumn{11}{|l|}{ HUMIRIACEAE } \\
\hline Endopleura uchi & $\begin{array}{l}\text { (Huber) } \\
\text { Cuatrec }\end{array}$ & 3 & 0.04 & 0.011 & 0.027 & 0.046 & 0.224 & 0.021 & 0.016 & 0.026 \\
\hline Sacoglottis guianensis & Benth & 7 & 0.09 & 0.026 & 0.055 & 0.093 & 0.458 & 0.042 & 0.034 & 0.053 \\
\hline \multicolumn{11}{|l|}{ HYPERICACEAE } \\
\hline Vismia baccifera & $\begin{array}{l}\text { (L) Triana } \\
\& \text { Planch }\end{array}$ & 136 & 1.81 & 0.501 & 0.274 & 0.463 & 1.689 & 0.155 & 0.328 & 0.373 \\
\hline Vismia cayennensis & (Aubl) DC & 246 & 3.28 & 0.907 & 0.123 & 0.208 & 3.396 & 0.311 & 0.609 & 0.475 \\
\hline Vismia guianensis & (Aubl) Pers & 66 & 0.88 & 0.243 & 0.110 & 0.185 & 0.921 & 0.084 & 0.164 & 0.171 \\
\hline \multicolumn{11}{|l|}{ LAMIACEAE } \\
\hline Vitex triflora & Vahl & 82 & 1.09 & 0.302 & 0.205 & 0.347 & 1.732 & 0.159 & 0.231 & 0.269 \\
\hline \multicolumn{11}{|l|}{ LAURACEAE } \\
\hline Aiouea myristicoides & Mez & 4 & 0.05 & 0.015 & 0.027 & 0.046 & 0.113 & 0.010 & 0.013 & 0.024 \\
\hline Aniba guianensis & Aubl & 55 & 0.73 & 0.203 & 0.247 & 0.417 & 2.191 & 0.201 & 0.202 & 0.273 \\
\hline $\begin{array}{l}\text { Dicypellium } \\
\text { caryophyllaceum }\end{array}$ & $\begin{array}{l}\text { (Mart) } \\
\text { Nees }\end{array}$ & 4 & 0.05 & 0.015 & 0.027 & 0.046 & 0.049 & 0.004 & 0.010 & 0.022 \\
\hline Dicypellium $s p$ & & 2 & 0.03 & 0.007 & 0.014 & 0.023 & 0.018 & 0.002 & 0.005 & 0.011 \\
\hline Endlicheria bracteolata & $\begin{array}{l}\text { (Meisn.) } \\
\text { C.K. Allen }\end{array}$ & 15 & 0.20 & 0.055 & 0.068 & 0.116 & 0.565 & 0.052 & 0.054 & 0.074 \\
\hline Endlicheria williamsii & O Schmidt & 8 & 0.11 & 0.029 & 0.027 & 0.046 & 0.297 & 0.027 & 0.028 & 0.034 \\
\hline Licaria guianensis & Aubl & 6 & 0.08 & 0.022 & 0.027 & 0.046 & 0.084 & 0.008 & 0.015 & 0.025 \\
\hline Mezilaurus itauba & $\begin{array}{l}\text { (Meisn) } \\
\text { Taub ex } \\
\text { Mez }\end{array}$ & 19 & 0.25 & 0.070 & 0.110 & 0.185 & 1.286 & 0.118 & 0.094 & 0.124 \\
\hline Mezilaurus lindaviana & $\begin{array}{l}\text { Schwacke } \\
\& \mathrm{Mez}\end{array}$ & 6 & 0.08 & 0.022 & 0.041 & 0.069 & 0.176 & 0.016 & 0.019 & 0.036 \\
\hline Nectandra cuspidata & Nees & 2 & 0.03 & 0.007 & 0.014 & 0.023 & 0.054 & 0.005 & 0.006 & 0.012 \\
\hline Nectandra pulverulenta & Nees & 20 & 0.27 & 0.074 & 0.110 & 0.185 & 0.799 & 0.073 & 0.073 & 0.111 \\
\hline Nectandra sp 1 & & 2 & 0.03 & 0.007 & 0.014 & 0.023 & 0.055 & 0.005 & 0.006 & 0.012 \\
\hline Ocotea canaliculata & (Rich) Mez & 203 & 2.71 & 0.748 & 0.479 & 0.810 & 6.255 & 0.573 & 0.661 & 0.711 \\
\hline Ocotea caudata & (Nees) Mez & 88 & 1.17 & 0.324 & 0.356 & 0.602 & 2.879 & 0.264 & 0.294 & 0.397 \\
\hline Ocotea cinerea & $\begin{array}{l}\text { van der } \\
\text { Werff }\end{array}$ & 16 & 0.21 & 0.059 & 0.041 & 0.069 & 0.296 & 0.027 & 0.043 & 0.052 \\
\hline Ocotea cujumary & Mart & 2 & 0.03 & 0.007 & 0.014 & 0.023 & 0.062 & 0.006 & 0.007 & 0.012 \\
\hline Ocotea glandulosa & Lasser & 2 & 0.03 & 0.007 & 0.014 & 0.023 & 0.076 & 0.007 & 0.007 & 0.012 \\
\hline Ocotea glomerata & (Nees) Mez & 2 & 0.03 & 0.007 & 0.014 & 0.023 & 0.114 & 0.010 & 0.009 & 0.014 \\
\hline Ocotea longifolia & Kunth & 4 & 0.05 & 0.015 & 0.027 & 0.046 & 0.079 & 0.007 & 0.011 & 0.023 \\
\hline $\begin{array}{l}\text { Rhodostemonodaphne } \\
\text { grandis }\end{array}$ & $\begin{array}{l}\text { (Mez) } \\
\text { Rohwer }\end{array}$ & 16 & 0.21 & 0.059 & 0.068 & 0.116 & 1.073 & 0.098 & 0.079 & 0.091 \\
\hline \multicolumn{11}{|l|}{ LECYTHIDACEAE } \\
\hline Bertholletia excelsa & Bonpl & 113 & 1.51 & 0.417 & 0.630 & 1.065 & 58.762 & 5.387 & 2.902 & 2.289 \\
\hline
\end{tabular}

$\mathrm{N}$.ind = number of individuals, $\mathrm{AD}=$ absolute density, $\mathrm{RD}=$ relative density, $\mathrm{AF}=$ absolute frequency, $\mathrm{RF}=$ relative frequency,

$\mathrm{ADo}=$ absolute dominance, $\mathrm{RDo}=$ relative dominance, $\mathrm{CV}=$ coverage value and $\mathrm{IV}=$ importance value . 
Table 1. Continued...

\begin{tabular}{|c|c|c|c|c|c|c|c|c|c|c|}
\hline FAMILY/SPECIES & AUTOR & N.ind & AD & RD & AF & RF & ADo & RDo & $\mathbf{C V}$ & IV \\
\hline Cariniana micrantha & Ducke & 1 & 0.01 & 0.004 & 0.014 & 0.023 & 1.611 & 0.148 & 0.076 & 0.058 \\
\hline Couratari guianensis & Aubl & 68 & 0.91 & 0.251 & 0.260 & 0.440 & 5.514 & 0.505 & 0.378 & 0.399 \\
\hline Couratari multiflora & (Sm) Eyma & 24 & 0.32 & 0.088 & 0.110 & 0.185 & 2.623 & 0.240 & 0.164 & 0.171 \\
\hline Couratari oblongifolia & $\begin{array}{l}\text { Ducke \& } \\
\text { Knuth }\end{array}$ & 3 & 0.04 & 0.011 & 0.014 & 0.023 & 0.214 & 0.020 & 0.015 & 0.018 \\
\hline Couratari stellata & A C Sm & 13 & 0.17 & 0.048 & 0.027 & 0.046 & 0.398 & 0.037 & 0.042 & 0.044 \\
\hline Eschweilera amazonica & R Knuth & 2 & 0.03 & 0.007 & 0.014 & 0.023 & 0.127 & 0.012 & 0.010 & 0.014 \\
\hline Eschweilera bracteosa & $\begin{array}{l}\text { (Poepp ex } \\
\text { O Berg) } \\
\text { Miers }\end{array}$ & 12 & 0.16 & 0.044 & 0.041 & 0.069 & 0.314 & 0.029 & 0.037 & 0.047 \\
\hline Eschweilera coriacea & $\begin{array}{l}(\mathrm{DC}) \mathrm{S} \text { A } \\
\text { Mori }\end{array}$ & 196 & 2.61 & 0.723 & 0.452 & 0.764 & 7.230 & 0.663 & 0.693 & 0.716 \\
\hline Eschweilera grandiflora & $\begin{array}{l}\text { (Aubl) } \\
\text { Sandwith }\end{array}$ & 8 & 0.11 & 0.029 & 0.068 & 0.116 & 0.471 & 0.043 & 0.036 & 0.063 \\
\hline Eschweilera pedicellata & $\begin{array}{l}\text { (Rich) S A } \\
\text { Mori }\end{array}$ & 22 & 0.29 & 0.081 & 0.082 & 0.139 & 0.503 & 0.046 & 0.064 & 0.089 \\
\hline Eschweilera sp & & 5 & 0.07 & 0.018 & 0.027 & 0.046 & 0.222 & 0.020 & 0.019 & 0.028 \\
\hline Gustavia augusta & $\mathrm{L}$ & 82 & 1.09 & 0.302 & 0.315 & 0.532 & 1.138 & 0.104 & 0.203 & 0.313 \\
\hline Gustavia hexapetala & (Aubl) Sm & 246 & 3.28 & 0.907 & 0.521 & 0.879 & 3.584 & 0.329 & 0.618 & 0.705 \\
\hline $\begin{array}{l}\text { Holopixidium } \\
\text { itacaiunensis }\end{array}$ & Pires & 213 & 2.84 & 0.785 & 0.301 & 0.509 & 5.488 & 0.503 & 0.644 & 0.599 \\
\hline Lecythis confertiflora & $\begin{array}{l}(\mathrm{A} C \mathrm{Sm}) \mathrm{S} \\
\text { A Mori }\end{array}$ & 2 & 0.03 & 0.007 & 0.014 & 0.023 & 0.033 & 0.003 & 0.005 & 0.011 \\
\hline Lecythis corrugata & Poit & 197 & 2.63 & 0.726 & 0.205 & 0.347 & 5.192 & 0.476 & 0.601 & 0.516 \\
\hline Lecythis holcogyne & $\begin{array}{l}\text { (Sandwith) } \\
\text { S A Mori }\end{array}$ & 4 & 0.05 & 0.015 & 0.014 & 0.023 & 0.076 & 0.007 & 0.011 & 0.015 \\
\hline Lecythis idatimon & Aubl & 78 & 1.04 & 0.288 & 0.068 & 0.116 & 2.140 & 0.196 & 0.242 & 0.200 \\
\hline Lecythis lurida & $\begin{array}{l}\text { (Miers) S A } \\
\text { Mori }\end{array}$ & 104 & 1.39 & 0.383 & 0.233 & 0.393 & 3.464 & 0.318 & 0.350 & 0.365 \\
\hline Lecythis pisonis & Cambess & 21 & 0.28 & 0.077 & 0.123 & 0.208 & 3.738 & 0.343 & 0.210 & 0.209 \\
\hline \multicolumn{11}{|l|}{ MALPIGHIACEAE } \\
\hline Byrsonima aerugo & Sagot & 21 & 0.28 & 0.077 & 0.055 & 0.093 & 0.658 & 0.060 & 0.069 & 0.077 \\
\hline Byrsonima chrysophylla & Kunth & 9 & 0.12 & 0.033 & 0.068 & 0.116 & 0.304 & 0.028 & 0.031 & 0.059 \\
\hline Byrsonima crispa & A Juss & 2 & 0.03 & 0.007 & 0.014 & 0.023 & 0.016 & 0.001 & 0.004 & 0.011 \\
\hline Byrsonima densa & (Poir) DC & 2 & 0.03 & 0.007 & 0.014 & 0.023 & 0.083 & 0.008 & 0.007 & 0.013 \\
\hline \multicolumn{11}{|l|}{ MALVACEAE } \\
\hline Apeiba echinata & Gaertn & 118 & 1.57 & 0.435 & 0.315 & 0.532 & 4.415 & 0.405 & 0.420 & 0.457 \\
\hline Apeiba glabra & Aubl & 4 & 0.05 & 0.015 & 0.027 & 0.046 & 0.164 & 0.015 & 0.015 & 0.025 \\
\hline Apeiba tibourbou & Aubl & 38 & 0.51 & 0.140 & 0.137 & 0.231 & 1.545 & 0.142 & 0.141 & 0.171 \\
\hline Ceiba pentandra & (L) Gaertn & 40 & 0.53 & 0.147 & 0.274 & 0.463 & 6.548 & 0.600 & 0.374 & 0.404 \\
\hline $\begin{array}{l}\text { Eriotheca } \\
\text { longipedicelata }\end{array}$ & $\begin{array}{l}\text { (Ducke) A } \\
\text { Robyns }\end{array}$ & 95 & 1.27 & 0.350 & 0.329 & 0.555 & 2.961 & 0.271 & 0.311 & 0.392 \\
\hline Guazuma ulmifolia & Lam & 283 & 3.77 & 1.043 & 0.466 & 0.787 & 12.724 & 1.166 & 1.105 & 0.999 \\
\hline Luehea grandiflora & $\begin{array}{l}\text { Mart \& } \\
\text { Zucc }\end{array}$ & 3 & 0.04 & 0.011 & 0.014 & 0.023 & 0.214 & 0.020 & 0.015 & 0.018 \\
\hline Matisia sp & & 28 & 0.37 & 0.103 & 0.219 & 0.370 & 3.606 & 0.331 & 0.217 & 0.268 \\
\hline Mollia lepidota & $\begin{array}{l}\text { Spruce ex } \\
\text { Benth }\end{array}$ & 72 & 0.96 & 0.265 & 0.068 & 0.116 & 3.825 & 0.351 & 0.308 & 0.244 \\
\hline Pachira aquatica & Aubl & 77 & 1.03 & 0.284 & 0.260 & 0.440 & 2.056 & 0.188 & 0.236 & 0.304 \\
\hline Patinoa paraensis & $\begin{array}{l}\text { (Huber) } \\
\text { Cuatrec }\end{array}$ & 121 & 1.61 & 0.446 & 0.110 & 0.185 & 5.961 & 0.546 & 0.496 & 0.393 \\
\hline Quararibea guianensis & Aubl & 392 & 5.23 & 1.445 & 0.342 & 0.579 & 5.672 & 0.520 & 0.983 & 0.848 \\
\hline Sterculia apelata & Ducke & 14 & 0.19 & 0.052 & 0.123 & 0.208 & 2.228 & 0.204 & 0.128 & 0.155 \\
\hline
\end{tabular}


Table 1. Continued...

\begin{tabular}{|c|c|c|c|c|c|c|c|c|c|c|}
\hline FAMILY/SPECIES & AUTOR & N.ind & AD & RD & $\mathbf{A F}$ & RF & ADo & RDo & $\mathrm{CV}$ & IV \\
\hline Sterculia excelsa & Mart & 7 & 0.09 & 0.026 & 0.068 & 0.116 & 0.599 & 0.055 & 0.040 & 0.065 \\
\hline Sterculia pruriens & $\begin{array}{l}\text { (Aubl) K } \\
\text { Schum }\end{array}$ & 183 & 2.44 & 0.675 & 0.438 & 0.741 & 9.601 & 0.880 & 0.777 & 0.765 \\
\hline $\begin{array}{l}\text { Theobroma } \\
\text { grandiflorum }\end{array}$ & $\begin{array}{l}\text { (Willd ex } \\
\text { Spreng) K } \\
\text { Schum }\end{array}$ & 18 & 0.24 & 0.066 & 0.068 & 0.116 & 0.383 & 0.035 & 0.051 & 0.072 \\
\hline Theobroma speciosum & $\begin{array}{l}\text { Willd ex } \\
\text { Spreng }\end{array}$ & 980 & 13.07 & 3.613 & 0.726 & 1.227 & 14.562 & 1.335 & 2.474 & 2.058 \\
\hline Theobroma subincanum & Mart & 53 & 0.71 & 0.195 & 0.164 & 0.278 & 0.947 & 0.087 & 0.141 & 0.187 \\
\hline \multicolumn{11}{|l|}{ MARCGRAVIACEAE } \\
\hline Norantea guianensis & Aubl & 2 & 0.03 & 0.007 & 0.014 & 0.023 & 0.034 & 0.003 & 0.005 & 0.011 \\
\hline \multicolumn{11}{|c|}{ MELASTOMATACEAE } \\
\hline Bellucia dichotoma & Cogn & 24 & 0.32 & 0.088 & 0.082 & 0.139 & 0.419 & 0.038 & 0.063 & 0.089 \\
\hline Bellucia grossularioides & (L) Triana & 236 & 3.15 & 0.870 & 0.260 & 0.440 & 5.613 & 0.515 & 0.692 & 0.608 \\
\hline Miconia affinis & DC & 10 & 0.13 & 0.037 & 0.041 & 0.069 & 0.109 & 0.010 & 0.023 & 0.039 \\
\hline Miconia minutiflora & $\begin{array}{l}\text { (Bonpl) } \\
\text { DC }\end{array}$ & 10 & 0.13 & 0.037 & 0.041 & 0.069 & 0.106 & 0.010 & 0.023 & 0.039 \\
\hline Miconia pyrifolia & Naudin & 58 & 0.77 & 0.214 & 0.219 & 0.370 & 1.618 & 0.148 & 0.181 & 0.244 \\
\hline Mouriri brachyanthera & Ducke & 22 & 0.29 & 0.081 & 0.055 & 0.093 & 0.450 & 0.041 & 0.061 & 0.072 \\
\hline \multicolumn{11}{|l|}{ MELIACEAE } \\
\hline Cedrela fissilis & Vell & 2 & 0.03 & 0.007 & 0.014 & 0.023 & 0.026 & 0.002 & 0.005 & 0.011 \\
\hline Cedrela odorata & $\mathrm{L}$ & 19 & 0.25 & 0.070 & 0.082 & 0.139 & 0.818 & 0.075 & 0.073 & 0.095 \\
\hline Guarea carinata & Ducke & 421 & 5.61 & 1.552 & 0.534 & 0.903 & 10.613 & 0.973 & 1.262 & 1.142 \\
\hline Guarea guidonia & $\begin{array}{l}\text { (L) } \\
\text { Sleumer }\end{array}$ & 34 & 0.45 & 0.125 & 0.137 & 0.231 & 1.703 & 0.156 & 0.141 & 0.171 \\
\hline Guarea kunthiana & A Juss & 346 & 4.61 & 1.276 & 0.438 & 0.741 & 7.618 & 0.698 & 0.987 & 0.905 \\
\hline Guarea silvatica & & 2 & 0.03 & 0.007 & 0.014 & 0.023 & 0.017 & 0.002 & 0.004 & 0.011 \\
\hline Guarea sp 1 & & 71 & 0.95 & 0.262 & 0.123 & 0.208 & 1.473 & 0.135 & 0.198 & 0.202 \\
\hline Trichilia cipo & $\begin{array}{l}\text { (A Juss) C } \\
\text { DC }\end{array}$ & 85 & 1.13 & 0.313 & 0.110 & 0.185 & 1.941 & 0.178 & 0.246 & 0.225 \\
\hline Trichilia lecointei & Ducke & 32 & 0.43 & 0.118 & 0.137 & 0.231 & 1.073 & 0.098 & 0.108 & 0.149 \\
\hline Trichilia micrantha & Benth & 126 & 1.68 & 0.464 & 0.274 & 0.463 & 2.569 & 0.236 & 0.350 & 0.388 \\
\hline Trichilia oligantha & $\mathrm{C} \mathrm{DC}$ & 4 & 0.05 & 0.015 & 0.014 & 0.023 & 0.055 & 0.005 & 0.010 & 0.014 \\
\hline Trichilia quadrijuga & Kunth & 24 & 0.32 & 0.088 & 0.082 & 0.139 & 0.542 & 0.050 & 0.069 & 0.092 \\
\hline \multicolumn{11}{|l|}{ MORACEAE } \\
\hline $\begin{array}{l}\text { Artocarpus } \\
\text { heterophyllus }\end{array}$ & Lam & 2 & 0.03 & 0.007 & 0.014 & 0.023 & 0.122 & 0.011 & 0.009 & 0.014 \\
\hline Artocarpus sp & Ducke & 12 & 0.16 & 0.044 & 0.055 & 0.093 & 0.126 & 0.012 & 0.028 & 0.049 \\
\hline Bagassa guianensis & Aubl & 7 & 0.09 & 0.026 & 0.041 & 0.069 & 0.319 & 0.029 & 0.028 & 0.041 \\
\hline Batocarpus amazonicus & $\begin{array}{l}\text { (Ducke) } \\
\text { Fosberg }\end{array}$ & 3 & 0.04 & 0.011 & 0.027 & 0.046 & 0.297 & 0.027 & 0.019 & 0.028 \\
\hline Brosimum acutifolium & Huber & 32 & 0.43 & 0.118 & 0.205 & 0.347 & 2.159 & 0.198 & 0.158 & 0.221 \\
\hline Brosimum guianense & $\begin{array}{l}\text { (Aubl) } \\
\text { Huber }\end{array}$ & 65 & 0.87 & 0.240 & 0.164 & 0.278 & 2.540 & 0.233 & 0.236 & 0.250 \\
\hline Brosimum lactescens & $\begin{array}{l}\text { (S Moore) } \\
\text { C C Berg }\end{array}$ & 42 & 0.56 & 0.155 & 0.082 & 0.139 & 1.594 & 0.146 & 0.150 & 0.147 \\
\hline Brosimum parinarioides & Ducke & 4 & 0.05 & 0.015 & 0.027 & 0.046 & 0.352 & 0.032 & 0.023 & 0.031 \\
\hline Brosimum potabile & Ducke & 8 & 0.11 & 0.029 & 0.068 & 0.116 & 0.491 & 0.045 & 0.037 & 0.063 \\
\hline Brosimum rubescens & Taub & 2 & 0.03 & 0.007 & 0.014 & 0.023 & 0.024 & 0.002 & 0.005 & 0.011 \\
\hline Clarisia racemosa & Ruiz \& Pav & 5 & 0.07 & 0.018 & 0.027 & 0.046 & 0.633 & 0.058 & 0.038 & 0.041 \\
\hline $\begin{array}{l}\text { Ficus americana subsp } \\
\text { guianensis }\end{array}$ & $\begin{array}{l}\text { (Desv.) } \\
\text { C.C. Berg }\end{array}$ & 6 & 0.08 & 0.022 & 0.055 & 0.093 & 0.512 & 0.047 & 0.035 & 0.054 \\
\hline
\end{tabular}

$\mathrm{N}$. ind $=$ number of individuals, $\mathrm{AD}=$ absolute density, $\mathrm{RD}=$ relative density, $\mathrm{AF}=$ absolute frequency, $\mathrm{RF}=$ relative frequency, $\mathrm{ADo}=$ absolute dominance, $\mathrm{RDo}=$ relative dominance, $\mathrm{CV}=$ coverage value and $\mathrm{IV}=$ importance value. 
Table 1. Continued...

\begin{tabular}{|c|c|c|c|c|c|c|c|c|c|c|}
\hline FAMILY/SPECIES & AUTOR & N.ind & AD & RD & $\mathbf{A F}$ & RF & ADo & RDo & $\mathrm{CV}$ & IV \\
\hline Ficus maxima & Mill & 25 & 0.33 & 0.092 & 0.123 & 0.208 & 4.486 & 0.411 & 0.252 & 0.237 \\
\hline Helicostylis pedunculata & Benoist & 22 & 0.29 & 0.081 & 0.096 & 0.162 & 0.424 & 0.039 & 0.060 & 0.094 \\
\hline Helicostylis scabra & $\begin{array}{l}\text { (J F } \\
\text { Macbr) C } \\
\text { C Berg } \\
\end{array}$ & 20 & 0.27 & 0.074 & 0.068 & 0.116 & 0.371 & 0.034 & 0.054 & 0.074 \\
\hline Helicostylis tomentosa & $\begin{array}{l}\text { (Poepp } \\
\text { \& Endl) } \\
\text { Rusby }\end{array}$ & 32 & 0.43 & 0.118 & 0.137 & 0.231 & 0.505 & 0.046 & 0.082 & 0.132 \\
\hline Maclura tinctoria & $\begin{array}{l}\text { (L) D Don } \\
\text { ex Steud }\end{array}$ & 23 & 0.31 & 0.085 & 0.055 & 0.093 & 0.612 & 0.056 & 0.070 & 0.078 \\
\hline Maquira calophylla & $\begin{array}{l}\text { (Poepp \& } \\
\text { Endl) C C } \\
\text { Berg }\end{array}$ & 6 & 0.08 & 0.022 & 0.027 & 0.046 & 0.081 & 0.007 & 0.015 & 0.025 \\
\hline Maquira coriacea & $\begin{array}{l}\text { (H Karst) } \\
\text { C C Berg }\end{array}$ & 13 & 0.17 & 0.048 & 0.068 & 0.116 & 0.231 & 0.021 & 0.035 & 0.062 \\
\hline Maquira guianensis & Aubl & 97 & 1.29 & 0.358 & 0.301 & 0.509 & 2.167 & 0.199 & 0.278 & 0.355 \\
\hline Maquira sclerophylla & $\begin{array}{l}\text { (Ducke) C } \\
\text { C Berg }\end{array}$ & 24 & 0.32 & 0.088 & 0.123 & 0.208 & 0.817 & 0.075 & 0.082 & 0.124 \\
\hline Naucleopsis caloneura & $\begin{array}{l}\text { (Huber) } \\
\text { Ducke }\end{array}$ & 27 & 0.36 & 0.100 & 0.096 & 0.162 & 0.359 & 0.033 & 0.066 & 0.098 \\
\hline Naucleopsis sp & & 2 & 0.03 & 0.007 & 0.014 & 0.023 & 0.023 & 0.002 & 0.005 & 0.011 \\
\hline Perebea mollis & $\begin{array}{l}\text { (Poepp } \\
\text { \& Endl) } \\
\text { Huber } \\
\text { subsp } \\
\text { mollis }\end{array}$ & 255 & 3.40 & 0.940 & 0.575 & 0.972 & 5.087 & 0.466 & 0.703 & 0.793 \\
\hline Pseudolmedia laevigata & Trecul & 18 & 0.24 & 0.066 & 0.055 & 0.093 & 0.446 & 0.041 & 0.054 & 0.067 \\
\hline Pseudolmedia laevis & $\begin{array}{l}\text { (Ruiz \& } \\
\text { Pav) J F } \\
\text { Macbr }\end{array}$ & 10 & 0.13 & 0.037 & 0.055 & 0.093 & 0.151 & 0.014 & 0.025 & 0.048 \\
\hline $\begin{array}{l}\text { Pseudolmedia } \\
\text { macrophylla }\end{array}$ & Trecul & 28 & 0.37 & 0.103 & 0.110 & 0.185 & 1.241 & 0.114 & 0.108 & 0.134 \\
\hline Sorocea duckei & $\begin{array}{l}\text { W C } \\
\text { Burger }\end{array}$ & 4 & 0.05 & 0.015 & 0.027 & 0.046 & 0.142 & 0.013 & 0.014 & 0.025 \\
\hline Sorocea guilleminiana & Gaudich & 11 & 0.15 & 0.041 & 0.082 & 0.139 & 0.175 & 0.016 & 0.028 & 0.065 \\
\hline $\begin{array}{l}\text { Trymatococcus } \\
\text { amazonicus }\end{array}$ & $\begin{array}{l}\text { Poepp \& } \\
\text { Endl }\end{array}$ & 14 & 0.19 & 0.052 & 0.096 & 0.162 & 0.160 & 0.015 & 0.033 & 0.076 \\
\hline \multicolumn{11}{|l|}{ MYRISTICACEAE } \\
\hline Iryanthera juruensis & Warb & 22 & 0.29 & 0.081 & 0.096 & 0.162 & 0.370 & 0.034 & 0.058 & 0.092 \\
\hline Iryanthera laevis & Markgr & 2 & 0.03 & 0.007 & 0.014 & 0.023 & 0.052 & 0.005 & 0.006 & 0.012 \\
\hline $\begin{array}{l}\text { Osteophloeum } \\
\text { platyspermum }\end{array}$ & $\begin{array}{l}\text { (Spruce } \\
\text { ex A DC) } \\
\text { Warb }\end{array}$ & 6 & 0.08 & 0.022 & 0.041 & 0.069 & 0.136 & 0.012 & 0.017 & 0.035 \\
\hline Virola crebrinervia & Ducke & 8 & 0.11 & 0.029 & 0.041 & 0.069 & 0.212 & 0.019 & 0.024 & 0.039 \\
\hline Virola elongata & $\begin{array}{l}\text { (Benth) } \\
\text { Warb }\end{array}$ & 18 & 0.24 & 0.066 & 0.082 & 0.139 & 0.271 & 0.025 & 0.046 & 0.077 \\
\hline Virola michelii & Heckel & 199 & 2.65 & 0.734 & 0.452 & 0.764 & 9.803 & 0.899 & 0.816 & 0.799 \\
\hline Virola pavonis & $\begin{array}{l}(\mathrm{A} \mathrm{DC}) \mathrm{A} \\
\mathrm{C} \mathrm{Sm}\end{array}$ & 14 & 0.19 & 0.052 & 0.055 & 0.093 & 0.741 & 0.068 & 0.060 & 0.071 \\
\hline Virola surinamensis & $\begin{array}{l}\text { (Rol ex } \\
\text { Rottb) } \\
\text { Warb } \\
\end{array}$ & 15 & 0.20 & 0.055 & 0.068 & 0.116 & 3.018 & 0.277 & 0.166 & 0.149 \\
\hline \multicolumn{11}{|l|}{ MYRTACEAE } \\
\hline Calyptranthes bipennis & O Berg & 67 & 0.89 & 0.247 & 0.205 & 0.347 & 1.150 & 0.105 & 0.176 & 0.233 \\
\hline
\end{tabular}


Table 1. Continued...

\begin{tabular}{|c|c|c|c|c|c|c|c|c|c|c|}
\hline FAMILY/SPECIES & AUTOR & N.ind & AD & RD & $\mathbf{A F}$ & RF & ADo & RDo & $\mathrm{CV}$ & IV \\
\hline Eugenia coffeifolia & $\mathrm{DC}$ & 2 & 0.03 & 0.007 & 0.014 & 0.023 & 0.048 & 0.004 & 0.006 & 0.012 \\
\hline Eugenia cupulata & Amshoff & 30 & 0.40 & 0.111 & 0.055 & 0.093 & 0.386 & 0.035 & 0.073 & 0.080 \\
\hline Eugenia flavescens & $\mathrm{DC}$ & 94 & 1.25 & 0.347 & 0.301 & 0.509 & 1.745 & 0.160 & 0.253 & 0.339 \\
\hline Eugenia patrisii & Vahl & 8 & 0.11 & 0.029 & 0.041 & 0.069 & 0.157 & 0.014 & 0.022 & 0.038 \\
\hline Eugenia ramiflora & Desv & 8 & 0.11 & 0.029 & 0.041 & 0.069 & 0.076 & 0.007 & 0.018 & 0.035 \\
\hline Myrcia fallax & $(\mathrm{Sw}) \mathrm{DC}$ & 34 & 0.45 & 0.125 & 0.192 & 0.324 & 0.626 & 0.057 & 0.091 & 0.169 \\
\hline Myrcia splendens & $(\mathrm{Sw}) \mathrm{DC}$ & 6 & 0.08 & 0.022 & 0.027 & 0.046 & 0.089 & 0.008 & 0.015 & 0.026 \\
\hline Psidium guajava & $\mathrm{L}$ & 10 & 0.13 & 0.037 & 0.055 & 0.093 & 0.114 & 0.010 & 0.024 & 0.047 \\
\hline$N I$ & $\mathrm{NI}$ & 7 & 0.09 & 0.026 & 0.027 & 0.046 & 0.429 & 0.039 & 0.033 & 0.037 \\
\hline \multicolumn{11}{|l|}{ NYCTAGINACEAE } \\
\hline Guapira hirsuta & $\begin{array}{l}\text { (Choisy) } \\
\text { Lundell }\end{array}$ & 25 & 0.33 & 0.092 & 0.082 & 0.139 & 0.502 & 0.046 & 0.069 & 0.092 \\
\hline Guapira venosa & $\begin{array}{l}\text { (Choisy) } \\
\text { Lundell }\end{array}$ & 819 & 10.92 & 3.019 & 0.699 & 1.180 & 19.578 & 1.795 & 2.407 & 1.998 \\
\hline Neea floribunda & $\begin{array}{l}\text { Poepp \& } \\
\text { Endl }\end{array}$ & 24 & 0.32 & 0.088 & 0.068 & 0.116 & 0.722 & 0.066 & 0.077 & 0.090 \\
\hline \multicolumn{11}{|l|}{ OLACACEAE } \\
\hline Cathedra acuminata & $\begin{array}{l}\text { (Benth) } \\
\text { Mier }\end{array}$ & 2 & 0.03 & 0.007 & 0.014 & 0.023 & 0.046 & 0.004 & 0.006 & 0.012 \\
\hline Chaunochiton kappleri & $\begin{array}{l}\text { (Sagot } \\
\text { ex Engl) } \\
\text { Ducke }\end{array}$ & 40 & 0.53 & 0.147 & 0.233 & 0.393 & 1.803 & 0.165 & 0.156 & 0.235 \\
\hline Heisteria acuminata & $\begin{array}{l}\text { (Humb } \\
\text { \& Bonpl) } \\
\text { Engl }\end{array}$ & 2 & 0.03 & 0.007 & 0.014 & 0.023 & 0.029 & 0.003 & 0.005 & 0.011 \\
\hline Heisteria barbata & Cuatrec & 2 & 0.03 & 0.007 & 0.014 & 0.023 & 0.078 & 0.007 & 0.007 & 0.013 \\
\hline Minquartia guianensis & Aubl & 18 & 0.24 & 0.066 & 0.096 & 0.162 & 0.903 & 0.083 & 0.075 & 0.104 \\
\hline \multicolumn{11}{|l|}{ OPILIACEAE } \\
\hline Agonandra brasiliensis & $\begin{array}{l}\text { Miers ex } \\
\text { Benth \& } \\
\text { Hook f }\end{array}$ & 14 & 0.19 & 0.052 & 0.082 & 0.139 & 0.275 & 0.025 & 0.038 & 0.072 \\
\hline \multicolumn{11}{|l|}{ PERACEAE } \\
\hline Pera cf glabrata & $\begin{array}{l}\text { (Schott) } \\
\text { Poepp. Ex } \\
\text { Baill } \\
\end{array}$ & 2 & 0.03 & 0.007 & 0.014 & 0.023 & 0.019 & 0.002 & 0.005 & 0.011 \\
\hline \multicolumn{11}{|l|}{ PHYLLANTHACEAE } \\
\hline $\begin{array}{l}\text { Discocarpus } \\
\text { essequeboensis }\end{array}$ & $\begin{array}{l}\text { Vahl ex } \\
\text { Vent }\end{array}$ & 10 & 0.13 & 0.037 & 0.041 & 0.069 & 0.176 & 0.016 & 0.027 & 0.041 \\
\hline $\begin{array}{l}\text { Hieronyma } \\
\text { alchorneoides }\end{array}$ & Allemao & 4 & 0.05 & 0.015 & 0.041 & 0.069 & 0.383 & 0.035 & 0.025 & 0.040 \\
\hline Margaritaria nobilis & $\mathrm{Lf}$ & 54 & 0.72 & 0.199 & 0.164 & 0.278 & 1.364 & 0.125 & 0.162 & 0.201 \\
\hline \multicolumn{11}{|l|}{ POLYGONACEAE } \\
\hline Coccoloba mollis & Casar & 42 & 0.56 & 0.155 & 0.219 & 0.370 & 1.304 & 0.120 & 0.137 & 0.215 \\
\hline Coccoloba ovata & Benth & 14 & 0.19 & 0.052 & 0.041 & 0.069 & 0.259 & 0.024 & 0.038 & 0.048 \\
\hline Triplaris weigeltiana & $\begin{array}{l}\text { (Rchb) } \\
\text { Kuntze }\end{array}$ & 6 & 0.08 & 0.022 & 0.041 & 0.069 & 0.112 & 0.010 & 0.016 & 0.034 \\
\hline \multicolumn{11}{|l|}{ PROTEACEAE } \\
\hline Roupala montana & Aubl & 23 & 0.31 & 0.085 & 0.151 & 0.255 & 1.399 & 0.128 & 0.107 & 0.156 \\
\hline \multicolumn{11}{|l|}{ QUIINACEAE } \\
\hline Lacunaria crenata & $\begin{array}{l}\text { (Tul) A C } \\
\text { Sm }\end{array}$ & 16 & 0.21 & 0.059 & 0.096 & 0.162 & 0.176 & 0.016 & 0.038 & 0.079 \\
\hline Quiina sp & & 2 & 0.03 & 0.007 & 0.014 & 0.023 & 0.022 & 0.002 & 0.005 & 0.011 \\
\hline RUBIACEAE & & & & & & & & & & \\
\hline
\end{tabular}


Table 1. Continued...

\begin{tabular}{|c|c|c|c|c|c|c|c|c|c|c|}
\hline FAMILY/SPECIES & AUTOR & N.ind & AD & RD & $\mathbf{A F}$ & RF & ADo & RDo & $\mathbf{C V}$ & IV \\
\hline Capirona decorticans & Spruce & 20 & 0.27 & 0.074 & 0.082 & 0.139 & 0.440 & 0.040 & 0.057 & 0.084 \\
\hline Chimarrhis turbinata & $\mathrm{DC}$ & 12 & 0.16 & 0.044 & 0.082 & 0.139 & 1.662 & 0.152 & 0.098 & 0.112 \\
\hline Cinchona sp & & 4 & 0.05 & 0.015 & 0.014 & 0.023 & 0.055 & 0.005 & 0.010 & 0.014 \\
\hline Cordiera macrophylla & $\begin{array}{l}\text { (K Schum) } \\
\text { Kuntze }\end{array}$ & 2 & 0.03 & 0.007 & 0.014 & 0.023 & 0.021 & 0.002 & 0.005 & 0.011 \\
\hline $\begin{array}{l}\text { Faramea } c f . \\
\text { involucellata }\end{array}$ & Müll.Arg. & 14 & 0.19 & 0.052 & 0.096 & 0.162 & 0.297 & 0.027 & 0.039 & 0.080 \\
\hline $\begin{array}{l}\text { Ferdinandusa } \\
\text { chlorantha }\end{array}$ & $\begin{array}{l}\text { (Wedd) } \\
\text { Standl }\end{array}$ & 24 & 0.32 & 0.088 & 0.068 & 0.116 & 0.840 & 0.077 & 0.083 & 0.094 \\
\hline Genipa americana & $\mathrm{L}$ & 13 & 0.17 & 0.048 & 0.055 & 0.093 & 0.360 & 0.033 & 0.040 & 0.058 \\
\hline Psychotria sp & $\mathrm{L}$ & 2 & 0.03 & 0.007 & 0.014 & 0.023 & 0.023 & 0.002 & 0.005 & 0.011 \\
\hline Randia armata & (Sw) DC & 2 & 0.03 & 0.007 & 0.014 & 0.023 & 0.046 & 0.004 & 0.006 & 0.012 \\
\hline \multicolumn{11}{|l|}{ RUTACEAE } \\
\hline Raputia sp & & 12 & 0.16 & 0.044 & 0.014 & 0.023 & 0.161 & 0.015 & 0.030 & 0.027 \\
\hline Zanthoxylum rhoifolium & Lam & 60 & 0.80 & 0.221 & 0.219 & 0.370 & 1.436 & 0.132 & 0.176 & 0.241 \\
\hline $\begin{array}{l}\text { Zanthoxylum } \\
\text { riedelianum }\end{array}$ & Engl & 77 & 1.03 & 0.284 & 0.247 & 0.417 & 2.265 & 0.208 & 0.246 & 0.303 \\
\hline Zanthoxylum sp 1 & & 6 & 0.08 & 0.022 & 0.027 & 0.046 & 0.094 & 0.009 & 0.015 & 0.026 \\
\hline \multicolumn{11}{|l|}{ SALICACEAE } \\
\hline Banara guianensis & Aubl & 14 & 0.19 & 0.052 & 0.027 & 0.046 & 0.154 & 0.014 & 0.033 & 0.037 \\
\hline Casearia grandiflora & Cambess & 4 & 0.05 & 0.015 & 0.027 & 0.046 & 0.043 & 0.004 & 0.009 & 0.022 \\
\hline Casearia javitensis & Kunth & 18 & 0.24 & 0.066 & 0.110 & 0.185 & 0.404 & 0.037 & 0.052 & 0.096 \\
\hline Casearia pitumba & Sleumer & 32 & 0.43 & 0.118 & 0.055 & 0.093 & 0.404 & 0.037 & 0.078 & 0.083 \\
\hline Casearia sylvestris & Sw & 4 & 0.05 & 0.015 & 0.014 & 0.023 & 0.063 & 0.006 & 0.010 & 0.015 \\
\hline Hasseltia floribunda & Kunth & 27 & 0.36 & 0.100 & 0.068 & 0.116 & 0.770 & 0.071 & 0.085 & 0.095 \\
\hline Homalium guianense & $\begin{array}{l}\text { (Aubl) } \\
\text { Oken }\end{array}$ & 14 & 0.19 & 0.052 & 0.041 & 0.069 & 0.140 & 0.013 & 0.032 & 0.045 \\
\hline Laetia procera & $\begin{array}{l}\text { (Poepp) } \\
\text { Eichler }\end{array}$ & 25 & 0.33 & 0.092 & 0.137 & 0.231 & 1.314 & 0.120 & 0.106 & 0.148 \\
\hline \multicolumn{11}{|l|}{ SAPINDACEAE } \\
\hline Cupania scrobiculata & Rich & 32 & 0.43 & 0.118 & 0.178 & 0.301 & 0.621 & 0.057 & 0.087 & 0.159 \\
\hline Matayba inelegans & $\begin{array}{l}\text { Spruce ex } \\
\text { Radlk }\end{array}$ & 46 & 0.61 & 0.170 & 0.082 & 0.139 & 0.617 & 0.057 & 0.113 & 0.122 \\
\hline Matayba $s p$ & Aubl & 106 & 1.41 & 0.391 & 0.110 & 0.185 & 1.784 & 0.164 & 0.277 & 0.246 \\
\hline Pseudima frutescens & $\begin{array}{l}\text { (Aubl) } \\
\text { Radlk }\end{array}$ & 30 & 0.40 & 0.111 & 0.137 & 0.231 & 0.469 & 0.043 & 0.077 & 0.128 \\
\hline Talisia longifolia & $\begin{array}{l}\text { (Benth) } \\
\text { Radlk }\end{array}$ & 12 & 0.16 & 0.044 & 0.068 & 0.116 & 0.160 & 0.015 & 0.029 & 0.058 \\
\hline Talisia mollis & $\begin{array}{l}\text { Kunth ex } \\
\text { Cambess }\end{array}$ & 2 & 0.03 & 0.007 & 0.014 & 0.023 & 0.029 & 0.003 & 0.005 & 0.011 \\
\hline Talisia veraluciana & Guarim & 4 & 0.05 & 0.015 & 0.027 & 0.046 & 0.083 & 0.008 & 0.011 & 0.023 \\
\hline Toulicia guianensis & Aubl & 69 & 0.92 & 0.254 & 0.205 & 0.347 & 1.091 & 0.100 & 0.177 & 0.234 \\
\hline Vouarana guianensis & Aubl & 2 & 0.03 & 0.007 & 0.014 & 0.023 & 0.022 & 0.002 & 0.005 & 0.011 \\
\hline \multicolumn{11}{|l|}{ SAPOTACEAE } \\
\hline $\begin{array}{l}\text { Chrysophyllum } \\
\text { argenteum }\end{array}$ & Jacq & 2 & 0.03 & 0.007 & 0.014 & 0.023 & 0.030 & 0.003 & 0.005 & 0.011 \\
\hline $\begin{array}{l}\text { Chrysophyllum } \\
\text { lucentifolium subsp } \\
\text { pachycarpum }\end{array}$ & $\begin{array}{l}\text { Pires \& T } \\
\text { D Penn }\end{array}$ & 106 & 1.41 & 0.391 & 0.384 & 0.648 & 4.923 & 0.451 & 0.421 & 0.497 \\
\hline Chrysophyllum prieurii & A DC & 4 & 0.05 & 0.015 & 0.027 & 0.046 & 0.053 & 0.005 & 0.010 & 0.022 \\
\hline $\begin{array}{l}\text { Chrysophyllum } \\
\text { venezuelanense }\end{array}$ & $\begin{array}{l}\text { (Pierre) } \mathrm{T} \\
\mathrm{D} \text { Penn }\end{array}$ & 6 & 0.08 & 0.022 & 0.027 & 0.046 & 0.470 & 0.043 & 0.033 & 0.037 \\
\hline Ecclinusa guianensis & Eyma & 31 & 0.41 & 0.114 & 0.068 & 0.116 & 1.239 & 0.114 & 0.114 & 0.115 \\
\hline
\end{tabular}


Table 1. Continued...

\begin{tabular}{|c|c|c|c|c|c|c|c|c|c|c|}
\hline FAMILY/SPECIES & AUTOR & N.ind & AD & RD & $\mathbf{A F}$ & RF & ADo & RDo & $\mathrm{CV}$ & IV \\
\hline Manilkara amazonica & $\begin{array}{l}\text { (Huber) A } \\
\text { Chev }\end{array}$ & 4 & 0.05 & 0.015 & 0.027 & 0.046 & 0.042 & 0.004 & 0.009 & 0.022 \\
\hline Manilkara huberi & Chevalier & 22 & 0.29 & 0.081 & 0.123 & 0.208 & 1.935 & 0.177 & 0.129 & 0.156 \\
\hline Micropholis acutangula & $\begin{array}{l}\text { (Ducke) } \\
\text { Eyma }\end{array}$ & 44 & 0.59 & 0.162 & 0.164 & 0.278 & 2.725 & 0.250 & 0.206 & 0.230 \\
\hline Micropholis egensis & $\begin{array}{l}\text { (A DC) } \\
\text { Pierre }\end{array}$ & 2 & 0.03 & 0.007 & 0.014 & 0.023 & 0.033 & 0.003 & 0.005 & 0.011 \\
\hline Micropholis guyanensis & $\begin{array}{l}\text { (A DC) } \\
\text { Pierre }\end{array}$ & 14 & 0.19 & 0.052 & 0.082 & 0.139 & 0.219 & 0.020 & 0.036 & 0.070 \\
\hline Micropholis venulosa & $\begin{array}{l}\text { (Mart \& } \\
\text { Eichler) } \\
\text { Pierre }\end{array}$ & 12 & 0.16 & 0.044 & 0.068 & 0.116 & 0.305 & 0.028 & 0.036 & 0.063 \\
\hline Pouteria anibifolia & $\begin{array}{l}\text { (Engl) } \\
\text { Eyma }\end{array}$ & 6 & 0.08 & 0.022 & 0.041 & 0.069 & 0.050 & 0.005 & 0.013 & 0.032 \\
\hline Pouteria anomala & $\begin{array}{l}\text { (Pires) T D } \\
\text { Penn }\end{array}$ & 5 & 0.07 & 0.018 & 0.027 & 0.046 & 0.120 & 0.011 & 0.015 & 0.025 \\
\hline Pouteria caimito & $\begin{array}{l}\text { (Ruiz \& } \\
\text { Pav) Radlk }\end{array}$ & 81 & 1.08 & 0.299 & 0.260 & 0.440 & 3.065 & 0.281 & 0.290 & 0.340 \\
\hline Pouteria cladantha & Sandwith & 35 & 0.47 & 0.129 & 0.164 & 0.278 & 1.264 & 0.116 & 0.122 & 0.174 \\
\hline Pouteria decorticans & T D Penn & 19 & 0.25 & 0.070 & 0.082 & 0.139 & 0.999 & 0.092 & 0.081 & 0.100 \\
\hline Pouteria elegans & $\begin{array}{l}\text { (A DC) } \\
\text { Baehni }\end{array}$ & 6 & 0.08 & 0.022 & 0.027 & 0.046 & 0.105 & 0.010 & 0.016 & 0.026 \\
\hline Pouteria engleri & Eyma & 6 & 0.08 & 0.022 & 0.041 & 0.069 & 0.173 & 0.016 & 0.019 & 0.036 \\
\hline Pouteria eugeniifolia & $\begin{array}{l}\text { (Pierre) } \\
\text { Baehni }\end{array}$ & 12 & 0.16 & 0.044 & 0.068 & 0.116 & 0.709 & 0.065 & 0.055 & 0.075 \\
\hline Pouteria filipes & Eyma & 33 & 0.44 & 0.122 & 0.192 & 0.324 & 1.517 & 0.139 & 0.130 & 0.195 \\
\hline Pouteria gongrijpii & Eyma & 68 & 0.91 & 0.251 & 0.151 & 0.255 & 1.873 & 0.172 & 0.211 & 0.226 \\
\hline Pouteria guianensis & Aubl & 105 & 1.40 & 0.387 & 0.370 & 0.625 & 3.048 & 0.279 & 0.333 & 0.430 \\
\hline Pouteria hispida & Eyma & 4 & 0.05 & 0.015 & 0.027 & 0.046 & 0.111 & 0.010 & 0.012 & 0.024 \\
\hline Pouteria jariensis & $\begin{array}{l}\text { Pires \& T } \\
\text { D Penn }\end{array}$ & 123 & 1.64 & 0.453 & 0.301 & 0.509 & 2.634 & 0.241 & 0.347 & 0.401 \\
\hline Pouteria krukovii & $\begin{array}{l}\text { (A C Sm) } \\
\text { Baehni }\end{array}$ & 2 & 0.03 & 0.007 & 0.014 & 0.023 & 0.017 & 0.002 & 0.004 & 0.011 \\
\hline Pouteria macrophylla & $\begin{array}{l}\text { (Lam) } \\
\text { Eyma }\end{array}$ & 160 & 2.13 & 0.590 & 0.397 & 0.671 & 5.564 & 0.510 & 0.550 & 0.590 \\
\hline Pouteria oppositifolia & $\begin{array}{l}\text { (Ducke) } \\
\text { Baehni }\end{array}$ & 8 & 0.11 & 0.029 & 0.055 & 0.093 & 0.318 & 0.029 & 0.029 & 0.050 \\
\hline Pouteria pachycarpa & $\begin{array}{l}\text { Pires \& T } \\
\text { D Penn }\end{array}$ & 16 & 0.21 & 0.059 & 0.096 & 0.162 & 0.238 & 0.022 & 0.040 & 0.081 \\
\hline Pouteria venosa & $\begin{array}{l}\text { (Mart) } \\
\text { Baehn }\end{array}$ & 30 & 0.40 & 0.111 & 0.082 & 0.139 & 0.690 & 0.063 & 0.087 & 0.104 \\
\hline Sarcaulus brasiliensis & $\begin{array}{l}\text { (A DC) } \\
\text { Eyma }\end{array}$ & 17 & 0.23 & 0.063 & 0.068 & 0.116 & 0.559 & 0.051 & 0.057 & 0.077 \\
\hline \multicolumn{11}{|l|}{ SIMAROUBACEAE } \\
\hline Picramnia sp & & 7 & 0.09 & 0.026 & 0.055 & 0.093 & 0.160 & 0.015 & 0.020 & 0.044 \\
\hline Simaba cedron & Planch & 170 & 2.27 & 0.627 & 0.370 & 0.625 & 2.900 & 0.266 & 0.446 & 0.506 \\
\hline Simaba guianensis & Aubl & 6 & 0.08 & 0.022 & 0.041 & 0.069 & 0.072 & 0.007 & 0.014 & 0.033 \\
\hline Simaba paraensis & Ducke & 3 & 0.04 & 0.011 & 0.027 & 0.046 & 0.330 & 0.030 & 0.021 & 0.029 \\
\hline Simarouba amara & Aubl & 72 & 0.96 & 0.265 & 0.301 & 0.509 & 4.995 & 0.458 & 0.362 & 0.411 \\
\hline \multicolumn{11}{|l|}{ SIPARUNACEAE } \\
\hline Siparuna guianensis & Aubl & 63 & 0.84 & 0.232 & 0.219 & 0.370 & 1.051 & 0.096 & 0.164 & 0.233 \\
\hline Siparuna $s p$ & & 25 & 0.33 & 0.092 & 0.110 & 0.185 & 0.463 & 0.042 & 0.067 & 0.107 \\
\hline SOLANACEAE & & & & & & & & & & \\
\hline
\end{tabular}


Table 1. Continued...

\begin{tabular}{|c|c|c|c|c|c|c|c|c|c|c|}
\hline FAMILY/SPECIES & AUTOR & N.ind & AD & RD & $\mathbf{A F}$ & RF & ADo & RDo & $\mathbf{C V}$ & IV \\
\hline Capsicum $s p$ & & 2 & 0.03 & 0.007 & 0.014 & 0.023 & 0.032 & 0.003 & 0.005 & 0.011 \\
\hline Solanum sendtnerianum & $\begin{array}{l}\text { Van } \\
\text { Heurck \& } \\
\text { Müll Arg }\end{array}$ & 4 & 0.05 & 0.015 & 0.014 & 0.023 & 0.036 & 0.003 & 0.009 & 0.014 \\
\hline \multicolumn{11}{|l|}{ ULMACEAE } \\
\hline Ampelocera edentula & Kuhlm & 72 & 0.96 & 0.265 & 0.301 & 0.509 & 3.182 & 0.292 & 0.279 & 0.355 \\
\hline \multicolumn{11}{|l|}{ URTICACEAE } \\
\hline Cecropia distachya & Huber & 211 & 2.81 & 0.778 & 0.219 & 0.370 & 12.786 & 1.172 & 0.975 & 0.773 \\
\hline Cecropia latiloba & Miq & 8 & 0.11 & 0.029 & 0.014 & 0.023 & 0.100 & 0.009 & 0.019 & 0.021 \\
\hline Cecropia membranacea & Trecul & 470 & 6.27 & 1.733 & 0.411 & 0.694 & 14.272 & 1.308 & 1.520 & 1.245 \\
\hline Cecropia obtusa & Trecul & 1000 & 13.33 & 3.687 & 0.630 & 1.065 & 26.376 & 2.418 & 3.052 & 2.390 \\
\hline Cecropia sciadophylla & Mart & 182 & 2.43 & 0.671 & 0.397 & 0.671 & 9.200 & 0.843 & 0.757 & 0.728 \\
\hline $\begin{array}{l}\text { Pourouma bicolor subsp } \\
\text { Digitata }\end{array}$ & $\begin{array}{l}\text { (Trecul) C } \\
\text { C Berg \& } \\
\text { Heusden }\end{array}$ & 19 & 0.25 & 0.070 & 0.123 & 0.208 & 0.986 & 0.090 & 0.080 & 0.123 \\
\hline Pourouma cecropiifolia & Mart & 22 & 0.29 & 0.081 & 0.110 & 0.185 & 0.681 & 0.062 & 0.072 & 0.110 \\
\hline Pourouma guianensis & Aubl & 128 & 1.71 & 0.472 & 0.342 & 0.579 & 6.381 & 0.585 & 0.528 & 0.545 \\
\hline Pourouma mollis & Trecul & 4 & 0.05 & 0.015 & 0.027 & 0.046 & 0.057 & 0.005 & 0.010 & 0.022 \\
\hline Urera caracasana & $\begin{array}{l}\text { (Jacq) } \\
\text { Griseb }\end{array}$ & 6 & 0.08 & 0.022 & 0.027 & 0.046 & 0.066 & 0.006 & 0.014 & 0.025 \\
\hline \multicolumn{11}{|l|}{ VIOLACEAE } \\
\hline Paypayrola grandiflora & Tul & 4 & 0.05 & 0.015 & 0.014 & 0.023 & 0.073 & 0.007 & 0.011 & 0.015 \\
\hline Rinorea guianensis & Aubl & 17 & 0.23 & 0.063 & 0.096 & 0.162 & 0.598 & 0.055 & 0.059 & 0.093 \\
\hline $\begin{array}{l}\text { Rinorea pubiflora var } \\
\text { pubiflora }\end{array}$ & $\begin{array}{l}\text { (Benth) } \\
\text { Sprague \& } \\
\text { Sandwith }\end{array}$ & 81 & 1.08 & 0.299 & 0.164 & 0.278 & 1.209 & 0.111 & 0.205 & 0.229 \\
\hline Rinorea riana & Kuntze & 10 & 0.13 & 0.037 & 0.055 & 0.093 & 0.095 & 0.009 & 0.023 & 0.046 \\
\hline \multicolumn{11}{|l|}{ VOCHYSIACEAE } \\
\hline Qualea dinizii & Ducke & 5 & 0.07 & 0.018 & 0.041 & 0.069 & 0.202 & 0.019 & 0.018 & 0.035 \\
\hline Ruizterania albiflora & $\begin{array}{l}\text { (Warm) } \\
\text { Marc Berti }\end{array}$ & 2 & 0.03 & 0.007 & 0.014 & 0.023 & 0.027 & 0.002 & 0.005 & 0.011 \\
\hline Vochysia obscura & Warm & 6 & 0.08 & 0.022 & 0.041 & 0.069 & 0.549 & 0.050 & 0.036 & 0.047 \\
\hline Vochysia sp & & 4 & 0.05 & 0.015 & 0.027 & 0.046 & 0.132 & 0.012 & 0.013 & 0.024 \\
\hline TOTAL & & 27126 & 361.7 & 100 & 59.19 & 100 & 1090.90 & 100 & 100 & 100 \\
\hline
\end{tabular}

$\mathrm{N}$. ind $=$ number of individuals, $\mathrm{AD}=$ absolute density, $\mathrm{RD}=$ relative density, $\mathrm{AF}=$ absolute frequency, $\mathrm{RF}=$ relative frequency, $\mathrm{ADo}=$ absolute dominance, $\mathrm{RDo}=$ relative dominance, $\mathrm{CV}=$ coverage value and $\mathrm{IV}=$ importance value.

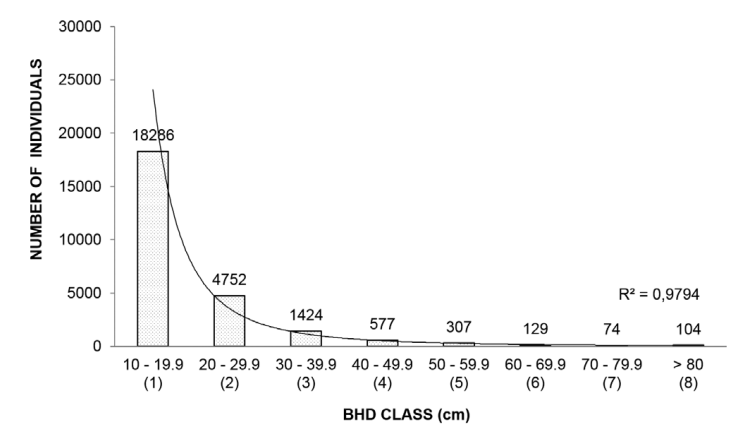

Figure 3. Diameter classes frequency distribution of sampled individuals in 75 ha of dense "terra firme" rainforest located in Belo Monte Hydroelectric Plant's area of influence, situated in Pará, Brazil. predominance of 10 to $19.9 \mathrm{~cm}$ diameter class individuals (71\%); $18.5 \%$ belongs to $20-29.9 \mathrm{~cm}$ class; $5.5 \% 30-39.9 \mathrm{~cm}$ class; others $(5.0 \%)$ belong to diametric classes above $50 \mathrm{~cm}$. The curve formed by established class values shows an "inverted J configuration" because there is a larger amount of smaller individuals and just emerging individuals.

The ten top species in terms of ecological Importance Value (IV) in descending order were: Alexa grandiflora (4.42), Cenostigma tocantinum (2.51), Cecropia obtusa (2.39), Bertholletia excelsa (2.29), Vouacapoua americana (2.27), Theobroma speciosum (2.06), Guapira venosa (1.99), Inga edulis (1.69), Jacaranda copaia (1.38) and Cecropia membranacea (1.24). 22.24\% of sampled individuals are concentrated in ten most important species in terms of ecological Importance Value (IV) which demonstrates their importance in the study area. The importance of 
most species can be mainly attributed to the high density of individuals. Only one of the ten species with the highest IV showed no high density value: Bertholletia excelsa.

Covered Values (CV) were similar to the IV order. There was only one difference: Vouacapoua americana was ranked third in covered value order and fourth in importance value.

\subsection{Sampling sufficience}

Linear regression with plateau response analysis determined among the number of species and sampling area the point of maximum inflection of the curve (X0) between the values of 41 and 42 ha. This value represents the minimum area required to satisfactorily express floristic richness. Therefore, 75 sampling units were satisfactory to represent the stretch of dense "terra firme" rainforest focused on in this study. The adjusted equations are defined by:

Line $\mathrm{Y}=133.1427+7.662 * \mathrm{X}($ when $\mathrm{X} \leq 41.2766)$

Plateau $Y=449.41$ (when $X>41.2766)$

$\mathrm{R}^{2}=95.46 \%$

where $\mathrm{Y}=$ species amount and $\mathrm{X}=$ sampling area (ha).

The survey sampling effort (75 plots) was approximately $44 \%$ greater than considered sufficient to express floristic composition by linear regression with plateau response model.

\section{Discussion}

According to results Fabaceae was the botanical family with the highest species richness (94). Condé and Tonini (2013) also reported the Fabaceae family as the one with more species in dense tropical rain forest in Northern Amazon. The greatest richness of Fabaceae's species corroborates other studies in Amazonia. Those other studies took place in the municipalities of Santa Bárbara do Pará-PA (Santos and Jardim, 2006), Cantá-RR (Silva, 2003) and $90 \mathrm{~km}$ from Manaus-AM (Oliveira et al., 2008).

Oliveira and Amaral (2004) found a large number of species from the Fabaceae, Sapotaceae and Lecythidaceae families in Central Amazonia "terra firme" forest. Sapotaceae and Lecythidaceae also presented high species richness in the present study with 35 species for the Lecythidaceae family and 21 for Sapotaceae.

Comparing the absolute total density studies with Condé and Tonini's studies (2013), it was found that the sampled area in the present study has a smaller value. This difference may be due to soil and topographic variations. Soil can act as a limiting factor (Mori et al., 1999). Species richness can vary depending on relief since in regions where the terrain is gentle, there is a lower species richness because the opportunities for niche specialisation get lower. However, this concept can only be confirmed through further studies that relate environmental factors and vegetation. In addition, inventories carried in the Amazon region differ in size and shape of plots, complicating comparisons between results of the several quantitative studies.

According to Oliveira et al. (2008) species with only one individual in sampled area are considered "locally rare". In the present study 16 locally rare species were found. It represents $3.4 \%$ of all the species found in 75 ha area. The percentage of $3.4 \%$ can be considered low compared to studies in the Amazon region, where we can mention the work done by Lima et al. (2012) which achieved 28\%. Higher percentages, ranging from 40 to $60 \%$, can be found in work in different Amazon regions such as the studies conducted by Oliveira and Amaral (2004), Oliveira and Amaral (2005) and Silva et al. (2008)

Just a few species predominated in importance value in sampled area considering the number of individuals (RD) and the basal area (RDo). This relationship was observed by Pires and Prance (1985). According to their study, the physiognomy of a stretch of forest depends heavily on the dominant species and the five or ten most common species usually represent more than half the number of Amazonian forest community. This observation was also verified by Melo (2004), Lima et al. (2007), Lima et al. (2012) and Condé and Tonini (2013).

According to Oliveira and Amaral (2004), estimated importance value for plant species can be used in management plans as an indicator of ecological importance due to the influence of the most frequent and dominant species in the basic flora's balance and maintenance of wildlife processes. It provides shelter and food.

The results for diameter structure show low amplitude along the diameter classes. There are a high number of trees at the beginning of the curve which can be explained by the strong presence of pioneer and opportunistic species. Additionally, diameter distribution was irregular in first classes. Therefore, it can be inferred that the plant community has experienced strong human or natural intervention reflected mainly in up to $30 \mathrm{~cm}$ diameter classes.

The diameter distribution "inverted J shaped" curve, demonstrates a greater amount of smaller individuals and few emerging individuals. This configuration was expected since distribution of size classes is a result of the forest dynamics, where the amount of space constricts the number of trees that can be accommodated in certain classes (Swaine, 1989).

Other studies in the region have used the same methodology and found a similar class of diameter distribution values (Salomão et al., 2007; MPEG, 2008). An accurate comparison was enabled due to the use of the same measurement criteria in all studies.

Diameter distribution analysis shows a common configuration in heterogeneous forests with diverse age trees, heterogeneous, and medium conservation condition. However, the large concentration of trees in class 1 may be related to human disturbance of the area. The forest gaps present in the region indicate an increase of pioneering and opportunistic species.

In general, the analysed plots had an average basal area (10 to $20 \mathrm{~m}^{2} / \mathrm{ha}$ ) lower than those registered for Amazon 
“terra firme" forests (30 to $40 \mathrm{~m}^{2}$ / ha) (Salomão et al., 2007). It is assumed this condition is related to anthropisation.

The UHE Belo Monte region has undergone considerable change from its original forest cover caused by the Trans-Amazon Highway's (BR-230) zone of influence and its transverse roads. Human occupation has been intensively induced by agrarian colonisation projects in recent years (Salomão et al., 2007). This interference leads to major changes in forest structure.

The floristic and phytosociological survey helped identify, in part, species distribution in the area. Floristic richness and its species diversity are considered high when compared to other studies conducted in nearby areas. This may be due because of ecotonal character as environmental heterogeneity.

\section{Acknowledgements}

This work was conducted in cooperation with Norte Energia SA and STCP Engenharia de Projetos LTDA running the PBA UHE Belo Monte. The authors thank these companies for allowing access to the areas and to the data obtained from field samples. The authors also thank all field staff for data collection: José Maria do Nascimento Jr., Edimar Almeida Campos; Carlos da Silva Rosário; Almir de Oliveira Gomes; Eva Moda de Vasconcelos.

\section{References}

Brasil. Eletrobrás, 2009. Aproveitamento hidrelétrico Belo Monte: estudo de impacto ambiental. Rio de Janeiro: Eletrobrás. 36 vol.

CONDÉ, TM. and TONINI, H., 2013. Fitossociologia de uma Floresta Ombrófila Densa na Amazônia Setentrional, Roraima, Brasil. Acta Amazonica, vol. 43, no. 3, p. 247-260. http://dx.doi. org/10.1590/S0044-59672013000300002.

HOPKINS, MJG., 2007. Modelling the known and unknown plant biodiversity of the Amazon basin. Journal of Biogeography, vol. 34, no. 8, p. 1400-1411. http://dx.doi.org/10.1111/j.13652699.2007.01737.x.

Instituto Brasileiro de Geografia e Estatística - IBGE, 2012. Manual técnico da vegetação brasileira. Rio de Janeiro: IBGE. 275 p. Manuais técnicos em geociências, no. 1.

LAURANCE, WF., 2008. Conserving the hottest of the hostspots. Biological Conservation, vol. 142, no. 6, p. 1137. http://dx.doi. org/10.1016/j.biocon.2008.10.011.

LIMA, AJN., TEIXEIRA, LM., CARNEIRO, VMC., SANTOS, J. and HIGUCHI, N., 2007. Análise da estrutura e do estoque de fitomassa de uma floresta secundária da região de Manaus AM, dez anos após corte raso seguido de fogo. Acta Amazonica, vol. 37, no. 1, p. 49-54. http://dx.doi.org/10.1590/S0044-59672007000100005.

LIMA, RBA., SILVA, JAA., MARANGON, LC., FERREIRA, RLC. and SILVA, RKS., 2012. Fitossociologia de um trecho de floresta ombrófila densa na Reserva de Desenvolvimento Sustentável Uacari, Carauari, Amazonas. Scientia Plena, vol. 8 , no. 1 , p. 1-12.

LIMA FILHO, DA., REVILLA, J., AMARAL, IL., MATOS, FDA., COÊLHO, LS., RAMOS, JF., SILVA, GB. and GUEDES, JO., 2004. Aspectos florísticos de 13 hectares da área de Cachoeira Porteira-PA. Acta Amazonica, vol. 34, no. 3, p. 415-423. http:// dx.doi.org/10.1590/S0044-59672004000300007.

MAGNUSSON, WE., LIMA, AP., LUIZÃO, R., LUIZÃO, F., COSTA, FRC., CASTILHO, CV. and KINUPP, VP., 2005. RAPELD: a modification of the Gentry method for biodiversity surveys in long-term ecological research sites. Biota Neotropica, vol. 5, no. 2, p. 1-6. http://dx.doi.org/10.1590/S1676-06032005000300002.

MELO, MS., 2004. Florística, fitossociologia e dinâmica de duas florestas secundárias antigas com histórias de uso diferentes no nordeste do Pará - Brasil. Piracicaba: Escola Superior de Agricultura Luíz de Queiroz, Universidade de São Paulo. 116 p. Dissertação de Mestrado em Recursos Florestais.

MORI, SA., RABELO, BV., TSOU, C. and DALY, D., 1999. Composition and structure of an eastern amazonian forest at Camaipi, Amapá, Brazil. Boletim do Museu Paraense Emílio Goeldi. Série Botânica, vol. 5, no. 1, p. 3-18.

MÜLLER-DOMBOIS, D. and ELLEMBERG, H., 1974. Aims and methods for vegetation ecology. New York: John Wiley \& Sons. $547 \mathrm{p}$.

Museu Paraense Emílio Goeldi - MPEG, 2008. Descrição e análise da flora da região do médio-baixo Rio Xingu. Belém: MPEG. 384 p.

OLIVEIRA, AN. and AMARAL, IL., 2004. Florística e fitossociologia de uma floresta de Vertente na Amazônia Central, Amazonas, Brasil. Acta Amazonica, vol. 34, no. 1, p. 21-34. http://dx.doi. org/10.1590/S0044-59672004000100004.

OLIVEIRA, AN. and AMARAL, IL., 2005. Aspectos florísticos, fitossociológicos e ecológicos de um sub-bosque de terra firme na Amazônia central, Amazonas, Brasil. Acta Amazonica, vol. 35, no. 1, p. 1-16. http://dx.doi.org/10.1590/S0044-59672005000100002.

OLIVEIRA, AN., AMARAL, IL., RAMOS, MBP., NOBRE, AD., COUTO, LB. and SAHDO, RM., 2008. Composição e diversidade florístico-estrutural de um hectare de floresta densa de terra firme na Amazônia Central, Amazonas, Brasil. Acta Amazonica, vol. 38, no. 4, p. 627-642. http://dx.doi.org/10.1590/ S0044-59672008000400005.

PIRES, JM. and PRANCE, GT. 1985. The vegetation types of the Brazilian Amazon. In PRANCE, G. and LOVEJOY, TE. (Eds.). Key environments Amazônia. New York: Pergamon Press. p. 109-145.

R Development Core Team, 2008. R: A Language and Environment for Statistical Computing. Vienna: The R Foundation for Statistical Computing. Available from: $<$ http://www.R-project.org/>. Access in: 5 May 2014

RAUNKIAER, C., 1934. The life form of plants and statistical plant geography. Oxford: Claredon. $632 \mathrm{p}$.

SALOMÃO, RP., VIEIRA, ICG., SUEMITSU, C., ROSA, NA., ALMEIDA, SS., AMARAL, DD. and MENEZES, MPM., 2007. As florestas de Belo Monte na grande curva do rio Xingu, Amazônia Oriental. Boletim do Museu Paraense Emilio Goeldi. Ciências Naturais, vol. 2, no. 3, p. 57-153.

SANTOS, GC. and JARDIM, MAG., 2006. Florística e estrutura do estrato arbóreo de uma floresta de várzea no Município de Santa Bárbara do Pará, Estado do Pará, Brasil. Acta Amazonica, vol. 36 , no. 4 , p. $437-446$. http://dx.doi.org/10.1590/S004459672006000400006 .

SILVA, USC., 2003. Fitossociologia do componente arbóreo e não arbóreo de uma Floresta Tropical em Cantá - RR. Belém: Universidade Federal Rural da Amazônia. 63 p. Dissertação de Mestrado em Ciências Florestais.

SILVA, KE., MATOS, FDA. and FERREIRA, MM., 2008. Composição florística e fitossociológica de espécies arbóreas do Parque Fenológico da Embrapa Amazônia Ocidental. Acta Amazonica, vol. 38, no. 2, p. 213-222. http://dx.doi.org/10.1590/ S0044-59672008000200004.

SWAINE, MD., 1989. Population dynamics of tree species in tropical forests. In HOLM-NIELSEN, LB., NIELSEN, IC. and BALSLEV, H. Tropical forests, botanical dynamics, speciation and diversity. Scotland: University of Aberdeen. p. 101-110. http:// dx.doi.org/10.1016/B978-0-12-353550-4.50016-2. 